\title{
Prevalence of anxiety and depression in South Asia during COVID-19:
}

\section{A systematic review and meta-analysis}

\author{
Md Mahbub Hossain ${ }^{1}$, Mariya Rahman ${ }^{1}$, Nusrat Fahmida Trisha ${ }^{1}$, Samia Tasnim ${ }^{1}$, Tasmiah Nuzhath ${ }^{1}$, \\ Nishat Tasnim Hasan ${ }^{1}$, Heather Clark ${ }^{1}$, Arindam Das ${ }^{2}$, E. Lisako J. McKyer ${ }^{1}$, Helal Uddin Ahmed ${ }^{3}$ Ping Ma ${ }^{1}$
}

\begin{abstract}
Introduction: The COVID-19 pandemic has impacted biopsychosocial health and wellbeing globally. Prepandemic studies suggest a high prevalence of common mental disorders, including anxiety and depression in South Asian countries, which may aggravate during this pandemic. This systematic metaanalytic review was conducted to estimate the pooled prevalence of anxiety and depression in South Asian countries during the COVID-19 pandemic.

Method: We systematically searched for cross-sectional studies on eight major bibliographic databases and additional sources up to October 12, 2020, that reported the prevalence of anxiety or depression in any of the eight South Asian countries. A random-effects model was used to calculate the pooled proportion of anxiety and depression.

Results: A total of 35 studies representing 41,402 participants were included in this review. The pooled prevalence of anxiety in 31 studies with a pooled sample of 28,877 was $41.3 \%$ (95\% confidence interval [CI]: 34.7-48.1, $I^{2}=99.18 \%$ ). Moreover, the pooled prevalence of depression was $34.1 \%$ (95\% CI: 28.9-39.4, $I^{2}$ =99\%) among 37,437 participants in 28 studies. Among the South Asian countries, India had a higher number of studies, whereas Bangladesh and Pakistan had a higher pooled prevalence of anxiety and depression. No studies were identified from Afghanistan, Bhutan, and Maldives. Studies in this review had high heterogeneity and varying prevalence across sub-groups.

Conclusion: South Asian countries have high prevalence rates of anxiety and depression, suggesting a heavy psychosocial burden during this pandemic. Clinical and public mental health interventions should be prioritized alongside improving the social determinants of mental health in these countries. Lastly, a low number of studies with high heterogeneity requires further research exploring the psychosocial epidemiology during COVID-19, which may inform better mental health policymaking and practice in South Asia.
\end{abstract}

Keywords: COVID-19; Mental Health; Anxiety; Depression; South Asia; Afghanistan; Bangladesh; Bhutan; India; Maldives; Nepal; Pakistan; Sri Lanka; Psychiatry; Epidemiology

\footnotetext{
${ }^{1}$ School of Public Health, Texas A\&M University, College Station, TX 77843, United States.

${ }^{2}$ Indian Institute of Health Management Research (IIHMR), Jaipur, Rajasthan 302029, India.

${ }^{3}$ National Institute of Mental Health (NIMH), Sher-E-Bangla Nagar, Dhaka 1207, Bangladesh.

* Corresponding author: Md Mahbub Hossain, School of Public Health, Texas A\&M University, College Station, TX 77843,

United States.Email: mhossain@tamu.edu
} 


\section{Introduction}

Novel coronavirus disease 2019 (COVID-19) is an acute respiratory illness caused by a newly discovered SARS-CoV-2 virus that emerged in December, $2019^{1,2}$. The rapid spread of this disease critically impacted health and wellbeing globally, which was declared as a pandemic by the World Health Organization (WHO) on March 11, $2020^{3}$. The sudden outburst of this highly infectious disease presented an unprecedented burden on mortality and morbidity across global nations ${ }^{1}$. In addition, healthcare systems and economies have been struggling to overcome the challenges imposed by this pandemic. To slow down the spread of this relatively unknown virus, countries have implemented several strategies such as quarantine, social distancing, stay at home orders, lockdowns, and border closures ${ }^{4,5}$. While the clinical care practitioners and public health experts have been focusing on containing the spread of the virus, the COVID-19 pandemic and related quarantine measures have taken a heavy toll on people's mental health and wellbeing ${ }^{6-9}$.

Several studies conducted on people's mental health during lockdown or isolation reported restriction of movement showed that when people are restricted to a certain kind of environment, their mental health is adversely affected ${ }^{10-13}$. Historically, quarantine has been related to psychological outcomes such as anxiety, depression, panic, irritability, somatic disorder, and insomnia ${ }^{14}$. These measures, as well as the fear and uncertainty related to the disease especially perceived susceptibility, treatment and control measures, exposure to misinformation in media regarding COVID-19, social isolation and loneliness, and economic hardships, have contributed to the adverse impact on the mental health of the population ${ }^{9,15}$. Previous studies also suggest that the emergency health crisis has a debilitating effect on the mental health of the general population ${ }^{16-18}$. For example, reports of depression, anxiety, panic attacks, as well as suicidal ideation increased during the SARS and Ebola outbreak ${ }^{19}$, where the reported rates of depression in the general population varied between $3 \%$ and $73.10 \%{ }^{20}$.

Recent studies have similarly shown that COVID-19 has affected mental health outcomes such as anxiety, depression, and post-traumatic stress symptoms ${ }^{6,7,9}$. Studies conducted in China in the early phase of COVID-19 found that the pandemic-affected individuals had a wide range of adverse psychological impacts of COVID-19 ${ }^{21-25}$. A recent meta-analysis by Salari et al., reported that the prevalence of stress $29.6 \%$, the prevalence of anxiety $31.9 \%$, and the prevalence of 
depression $33.7 \%$ globally ${ }^{6}$. Health professionals, older adults, and those with preexisting psychological disorders, especially vulnerable to these poor mental health outcomes ${ }^{7,9}$.

Developing countries, especially countries in South Asia, are already struggling to combat the high mortality and morbidity rates caused by COVID-19 due to a highly dense population and low-resource settings ${ }^{26}$. In such situations, the mental health service takes a backseat even though there has been an increase in the demand for mental health services ${ }^{27,28}$. Despite substantial evidence, there has been negligence in identifying people with mental health illnesses who have been impacted by the pandemic, especially in resource-poor countries ${ }^{29,30}$. This is manifested in the lack of mental health service availability in developing countries during this outbreak. According to the World Health Organization, $80 \%$ of the developed countries are offering mental health services using telemedicine and teletherapy, whereas less than $50 \%$ of the developing countries are providing such services during the pandemic ${ }^{31}$.

In South Asia, pre-pandemic studies suggest a high burden of mental health disorders in this region with limited access to mental health services ${ }^{32-35}$. Previous systematic reviews of the prevalence of mental health disorder in South Asian countries suggest high prevalence rates for mental disorders, including depression, anxiety, mood disorders, suicidal behavior and selfharm, schizophrenia, substance use disorders, and other mental health problems ${ }^{36,37}$. However, there is a lack of synthesized evidence on the regional and country-level estimates on the burden of common mental disorders such as anxiety and depression during the pandemic, where 150-200 million people in this region were suffering from mental disorders prior to COVID-19 pandemic ${ }^{38}$.

We aimed to address this knowledge gap and conducted a quantitative systematic review to estimate the pooled prevalence of anxiety and depression in South Asia during COVID-19. The evidence synthesized in this review will provide an overview of the prevalence of depression and anxiety, which may inform better decision-making and future research on mental health in South Asia. 


\section{Methodology}

This systematic review was conducted in accordance with the Preferred Reporting Items for Systematic Reviews and Meta-Analyses (PRISMA) statement ${ }^{39}$.

\subsection{Data sources and search strategy}

We conducted a comprehensive literature search in Medline, Embase, American Psychological Association (APA) PsycInfo, Academic Search Ultimate, Health Source Nursing/Academic Edition, Health Policy Reference Center, Cumulative Index to Nursing and Allied Health Literature (CINAHL), and Web of Science databases using the search query provided in Table 1. In each database, those keywords were searched using Boolean operators within the titles, abstracts, keywords, and subject headings (for example, MeSH terms). As this review aimed to focus on COVID-19, the search timeframe was set between 2019 and 2020. The preliminary search was conducted on August 16, 2020, and updated on October 12, 2020. In addition, we searched Google Scholar and reached out to mental health researchers in South Asia to identify relevant articles. Lastly, we also searched the bibliographies references of relevant articles to find additional studies that may fit this review.

\subsection{Selection criteria}

We included studies if they met the following criteria:

a) cross-sectional by design,

b) reported the prevalence of depression or anxiety during COVID-19,

c) measured depression or anxiety using any validated measurement tools or scales,

d) included participants from any of the eight South Asian countries, and

e) published as peer-reviewed articles in the English language. 
Table 1: Search strategy used in this systematic review and meta-analysis

\begin{tabular}{|c|c|c|}
\hline $\begin{array}{l}\text { Search } \\
\text { query }\end{array}$ & Search topic & $\begin{array}{l}\text { Search keywords (titles, abstracts, and subject headings) } \\
\text { with Boolean operators }\end{array}$ \\
\hline 1 & $\begin{array}{l}\text { Exposure/ } \\
\text { Context }\end{array}$ & $\begin{array}{l}\text { "Coronavirus" OR "COVID-19" OR "SARS-CoV-2" OR “2019- } \\
\text { nCoV" }\end{array}$ \\
\hline 2 & $\begin{array}{l}\text { Outcome of } \\
\text { interest }\end{array}$ & $\begin{array}{l}\text { "Depression" OR "Depressive symptoms" OR "Depressive } \\
\text { disorder*” OR “Anxiety" OR “Social Anxiety” or "Social } \\
\text { Phobia" OR "Anxiety disorder*” }\end{array}$ \\
\hline 3 & $\begin{array}{l}\text { Epidemiological } \\
\text { phenomenon }\end{array}$ & $\begin{array}{l}\text { "Prevalence" OR "Incidence" OR "rate*" OR "ratio*" OR } \\
\text { "Epidemiolog*” OR "risk factor*” OR "relative risk" OR "odds } \\
\text { ratio" OR "risk ratio" OR "disease burden" }\end{array}$ \\
\hline 4 & $\begin{array}{l}\text { Population of } \\
\text { interest }\end{array}$ & $\begin{array}{l}\text { "Afghan*" OR "Bangladesh*” OR "Bhutan*” OR "India*” OR } \\
\text { "Maldiv*” OR "Nepal*” OR "Pakistan*” OR "Sri Lanka*” OR } \\
\text { "South Asia*” }\end{array}$ \\
\hline $\begin{array}{l}\text { Final } \\
\text { search } \\
\text { query }\end{array}$ & $\begin{array}{l}\text { Intersection of } \\
\text { four topics }\end{array}$ & 1 AND 2 AND 3 AND 4 \\
\hline
\end{tabular}

We excluded articles that did not meet any of these primary criteria. Also, we considered original articles, short communications, and research letters that reported the methodology of the respective studies. Therefore, review articles or commentaries that did not present empirical findings with methodology were excluded from this review. Moreover, we excluded preprints that were not published as peer-reviewed journal articles. Also, we excluded studies that included people with pre-existing mental disorders or specialized population groups (for example, prison population), or focused on South Asian participants living abroad. Such studies were excluded as they may not reflect the burden of depression or anxiety during COVID-19 among people living in the South Asian region. 


\subsection{Study selection}

Two authors independently reviewed the titles and abstracts of the retrieved studies. At the end of the primary screening, conflicts in terms of eligibility of any citation were addressed through discussion with a third author. Citations that appeared to be eligible were selected for the fulltext evaluation. Further, full-text articles meeting all criteria of this review were retained for data extraction and subsequent analyses.

\subsection{Data extraction}

A data extraction form was prepared in Microsoft Excel to extract and record data-fields from the finally recruited articles. Data on the following variables were extracted: author information, year of publication, study design, response rate, recruitment strategy, sampling method, sample size and socio-demographic characteristics, assessment tools with cut-off values, and the prevalence of anxiety or depression in the respective study. Two authors independently extracted data from each article. Two separate datasets were reviewed and evaluated to unify addressing potential inconsistencies by another author at the end of the data extraction process.

\subsection{Quality assessment of the reviewed studies}

In this review, we used a modified version of the Newcastle-Ottawa Quality Assessment Scale adapted for cross-sectional studies, which has been used in similar meta-analyses previously ${ }^{7,40}$. This scale assessed the quality of cross-sectional studies in the following domains:

1) representativeness of the study (whether the study included all eligible participants or used random sampling approaches ensuring the representativeness of the study population),

2) justified sample size (provided justification for the chosen sample size through theoretical and statistical measures),

3) response rate (equal or more than $80 \%$ ),

4) used validated measurement tools with appropriate cut-offs, 
5) reported adequate statistics for the study findings.

A positive response in each domain could receive 1 point; thus, the total score could range from 0 to 5 on this modified scale. Studies receiving equal or more than 3 points were graded to have a low risk of bias, whereas studies with less than 3 points were regarded to have a high risk of bias according to this scale.

\subsection{Statistical analyses}

We performed meta-analyses using Open MetaAnalyst ${ }^{41}$ and StatsDirect ${ }^{42}$ software packages. First, we transformed the proportion values from individual studies using the Freeman-Tukey double arcsine method for prevalence pooling, which prevented the variance instability for prevalence values near $0 \%$ or $100 \%{ }^{43}$. Moreover, this method suppressed the confidence intervals (CI) from extending beyond $0 \%$ and $100 \%$. Further, we used a generic inverse-variance method with a random-effects model to calculate the pooled prevalence from multiple studies ${ }^{44}$. The Cochrane's Q-test was used to assess heterogeneity at the significance level of $p<.1$. Also, we used the $I^{2}$ statistics to categorize heterogeneity as low (25\% to $50 \%$ ), moderate (51\% to $75 \%$ ), and high (above $75 \%)^{45}$.

\subsection{Subgroup analyses}

Subgroup analyses are helpful to assess between-group variations of the prevalence as well as examine potential sources of heterogeneity across studies. We performed subgroup analyses by country, gender, populations, quality of the studies, measurement scale, and severity of depression and anxiety.

\subsection{Meta-regression}

We conducted meta-regression analyses to evaluate the correlations between the study level covariates and the pooled prevalence estimates. We used the mean age, quality of the studies, and the percentage of female participants in meta-regression models. In this meta-analytic 
review, factors were included in multivariate meta-regression analyses if they were significant at $p<.2$ level in univariate models.
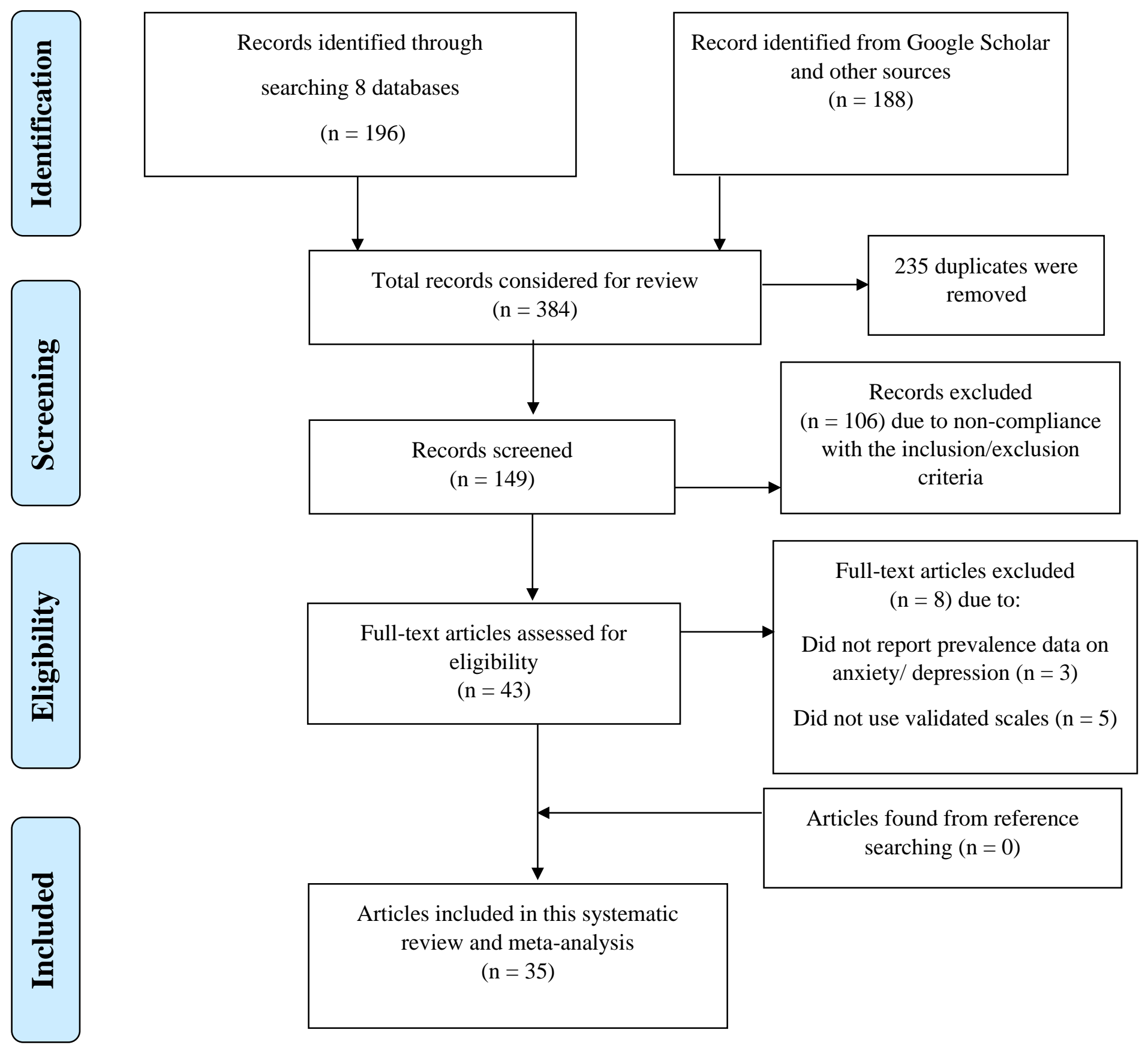

Figure 1: Flow diagram of the literature search process 


\subsection{Sensitivity analyses}

Sensitivity analyses were performed in this meta-analysis to assess the influence of individual studies on the pooled prevalence estimates or heterogeneity. We excluded each study and calculated the pooled prevalence and $I^{2}$ statistics for the rest of the studies.

\subsection{Evaluation of the publication bias}

We visually inspected the funnel plots and conducted Egger's regression tests to evaluate the publication bias that may exist and affect the generalizability of the study findings. A $p$ value less than 0.1 was considered as an indication of publication bias in Egger's test.

\section{Results}

A PRISMA flowchart detailing the literature review process is illustrated in Figure 1. We found a total of 384 citations from selected databases and additional sources. After excluding 235 duplicates, we examined the titles and abstracts of 149 citations using the pre-determined eligibility criteria. At the end of the preliminary screening process, 43 citations were selected for full-text evaluation. Eight articles were excluded that did not meet all criteria. Finally, the remaining 35 articles representing 41,402 participants were retained in this meta-analytic review ${ }^{46-80}$.

\subsection{Characteristics of the included studies}

An overview of the included studies is provided in Table 2. The highest number of studies were conducted in India ( $(n=19)$, followed by Bangladesh $(n=7)$, Pakistan $(n=5)$, Nepal $(n=3)$, and Sri Lanka $(\mathrm{n}=1)$. No studies were identified from Afghanistan, Bhutan, and the Maldives. Among the included studies, the sample size ranged from 41 to 10,178. Most studies recruited young adult participants with a mean age of the participants ranging from 25.75 to 42.5 years. The median percentage of female participants was $45.4 \%$ among the included studies. Moreover, most studies $(n=29)$ used online platforms, including email and social media sites, to recruit participants and conduct surveys. Response rates ranged from 33.5\% to 97.4\% across individual studies. Most studies $(n=18)$ in this review recruited healthcare providers as study participants. Two studies recruited patients, and the remaining 15 studies consisted of general population with different demographic and socioeconomic conditions. 
Table 2: Characteristics of the studies included in this meta-analytic review

\begin{tabular}{|c|c|c|c|c|c|c|c|c|c|}
\hline $\begin{array}{l}\text { Authors } \\
\text { and } \\
\text { publication } \\
\text { year }\end{array}$ & Country & $\begin{array}{l}\text { Study } \\
\text { design; } \\
\text { response } \\
\text { rate }\end{array}$ & $\begin{array}{l}\text { Recruitment } \\
\text { strategy; } \\
\text { sampling } \\
\text { method }\end{array}$ & $\begin{array}{l}\text { Sample size } \\
\text { and } \\
\text { characteristics }\end{array}$ & $\begin{array}{l}\text { Mean } \\
\text { age } \\
\text { with } \\
\text { SD }\end{array}$ & $\begin{array}{l}\text { Female } \\
(\%)\end{array}$ & $\begin{array}{l}\text { Education and } \\
\text { occupation }\end{array}$ & $\begin{array}{l}\text { Assessment } \\
\text { scale/tools } \\
\text { and cut-off } \\
\text { values }\end{array}$ & $\begin{array}{l}\text { Prevalence of } \\
\text { anxiety/ } \\
\text { depression }\end{array}$ \\
\hline $\begin{array}{l}\text { Ahmad et } \\
\text { al. (2020) }\end{array}$ & India & $\begin{array}{l}\text { Cross- } \\
\text { sectional; } \\
\text { NR }\end{array}$ & $\begin{array}{l}\text { Online; } \\
\text { random }\end{array}$ & $\begin{array}{l}\text { 392; general } \\
\text { population }\end{array}$ & $\begin{array}{l}30.3 \\
( \pm 9.28)\end{array}$ & 47.2 & $\begin{array}{l}\text { Graduate and } \\
\text { above } 91.9 ; \text { student } \\
41.3 \% \text {, Service } \\
\text { holders } 43.2 \%\end{array}$ & GAD-7 (>10) & Anxiety: $25.3 \%$ \\
\hline $\begin{array}{l}\text { Ahmed et } \\
\text { al. (2020) }\end{array}$ & Bangladesh & $\begin{array}{l}\text { Cross- } \\
\text { sectional; } \\
\text { NR }\end{array}$ & Online; NR & $\begin{array}{l}\text { 500; general } \\
\text { population }\end{array}$ & 25.95 & 32.3 & $\begin{array}{l}\text { Student } 65.3 \% \text {, } \\
\text { service holders } \\
25 \%\end{array}$ & $\begin{array}{l}\text { DASS-21 } \\
\text { (Anxiety }>4, \\
\text { Depression } \\
>5 \text { ) }\end{array}$ & $\begin{array}{l}\text { Anxiety: } 39.8 \% \text {; } \\
\text { Depression: } 43 \%\end{array}$ \\
\hline $\begin{array}{l}\text { Amin et al. } \\
(2020)\end{array}$ & Pakistan & $\begin{array}{l}\text { Cross- } \\
\text { sectional; } \\
\text { NR }\end{array}$ & Online; NR & $\begin{array}{l}389 ; \text { healthcare } \\
\text { providers }\end{array}$ & $\begin{array}{l}35 \\
\text { (Media } \\
\text { n) }\end{array}$ & 48.33 & $\begin{array}{l}\text { Physician trainees } \\
74.3 \% \text {, post- } \\
\text { graduate } \\
\text { qualification } \\
25.7 \% \text {; front-line } \\
\text { physicians }\end{array}$ & SRQ- $20(>8)$ & $\begin{array}{l}\text { Anxiety/ } \\
\text { depression: } 43 \%\end{array}$ \\
\hline $\begin{array}{l}\text { Banna et al. } \\
(2020)\end{array}$ & Bangladesh & $\begin{array}{l}\text { Cross- } \\
\text { sectional; } \\
\text { NR }\end{array}$ & $\begin{array}{l}\text { Online; } \\
\text { convenience } \\
\text { sampling }\end{array}$ & $\begin{array}{l}\text { 1427; general } \\
\text { population }\end{array}$ & 25.75 & 28.5 & $\begin{array}{l}\text { Undergraduate } \\
59 \% \text { and graduate } \\
28.3 \% \text {; students } \\
43.7 \% \text {, Service } \\
\text { holders } 42.2 \%\end{array}$ & $\begin{array}{l}\text { DASS-21 } \\
\text { (Anxiety >6, } \\
\text { Depression } \\
>9 \text { ) }\end{array}$ & $\begin{array}{l}\text { Anxiety: } 57.9 \% \text {; } \\
\text { Depression: } \\
33.7 \%\end{array}$ \\
\hline $\begin{array}{l}\text { Chatterjee } \\
\text { et al. (2020) }\end{array}$ & India & $\begin{array}{l}\text { Cross- } \\
\text { sectional; } \\
\text { NR }\end{array}$ & Online; NR & $\begin{array}{l}\text { 152; healthcare } \\
\text { providers }\end{array}$ & $\begin{array}{l}42.05 \\
( \pm 12.19)\end{array}$ & 21.7 & $\begin{array}{l}\text { Post-graduate } \\
63.2 \% \text { and } \\
\text { graduate } 34.2 \% \text {; } \\
\text { Government } \\
\text { doctor } 66.4 \% \text {, Non- } \\
\text { government } \\
\text { doctors } 33.6 \%\end{array}$ & DASS-21 & $\begin{array}{l}\text { Anxiety: } 39.5 \% \text {; } \\
\text { Depression: } \\
34.9 \%\end{array}$ \\
\hline $\begin{array}{l}\text { Chew et al. } \\
(2020) a\end{array}$ & India & $\begin{array}{l}\text { Cross- } \\
\text { sectional; } \\
33.5 \%\end{array}$ & NR & $\begin{array}{l}384 ; \text { healthcare } \\
\text { provider }\end{array}$ & 27.7 & 65.4 & $\begin{array}{l}\text { physicians } 21.95 \text {, } \\
\text { nurses } 43.5 \% \text {, } \\
\text { other non-medical } \\
\text { staff } 10.8 \% \text {. }\end{array}$ & $\begin{array}{l}\text { DASS-21 } \\
\text { (Anxiety >7, } \\
\text { Depression } \\
>9 \text { ) }\end{array}$ & $\begin{array}{l}\text { Anxiety/ } \\
\text { depression } 0.8 \%\end{array}$ \\
\hline $\begin{array}{l}\text { Chew et al. } \\
(2020) b\end{array}$ & India & $\begin{array}{l}\text { Cross- } \\
\text { sectional; } \\
90.6 \%\end{array}$ & NR & $\begin{array}{l}\text { 426; healthcare } \\
\text { provider }\end{array}$ & 29 & 64.3 & $\begin{array}{l}\text { Mostly physicians } \\
\text { and Nurses }\end{array}$ & $\begin{array}{l}\text { DASS-21 } \\
\text { (Anxiety >7, }\end{array}$ & $\begin{array}{l}\text { Anxiety: } 17.1 \% \text {; } \\
\text { Depression: } \\
12.4 \%\end{array}$ \\
\hline
\end{tabular}




\begin{tabular}{|c|c|c|c|c|c|c|c|c|c|}
\hline & & & & & & & & $\begin{array}{l}\text { Depression } \\
>9)\end{array}$ & \\
\hline $\begin{array}{l}\text { Desai et al. } \\
(2020)\end{array}$ & India & $\begin{array}{l}\text { Cross- } \\
\text { sectional; } \\
\text { NR }\end{array}$ & $\begin{array}{l}\text { Online; } \\
\text { snowball } \\
\text { sampling }\end{array}$ & $\begin{array}{l}\text { 1537; general } \\
\text { population }\end{array}$ & $\begin{array}{l}77.7 \% \\
\text { below } \\
30 \\
\text { years }\end{array}$ & 50 & $\begin{array}{l}\text { Undergraduate } \\
61.9 \% \text {; students } \\
67.5 \% \text {, working } \\
\text { professionals } \\
32.5 \%\end{array}$ & $\begin{array}{l}\text { GAD-7 } \\
(\text { Anxiety >5); } \\
\text { PHQ-9 } \\
\text { (Depression } \\
>5 \text { ) }\end{array}$ & $\begin{array}{l}\text { Anxiety: } 41.5 \% \text {; } \\
\text { Depression } 47 \%\end{array}$ \\
\hline $\begin{array}{l}\text { Grover et } \\
\text { al. (2020) }\end{array}$ & India & $\begin{array}{l}\text { Cross- } \\
\text { sectional; } \\
90.05 \%\end{array}$ & Online; NR & $\begin{array}{l}\text { 1685; general } \\
\text { population }\end{array}$ & 41.26 & 36.3 & $\begin{array}{l}\text { Graduate and } \\
\text { above } 93.8 \% \text {; } \\
\text { healthcare worker } \\
47.1 \% \text {, Service } \\
\text { holder } 11.4 \% \text {, } \\
\text { Businessperson } \\
\text { 10\%, Student } 0.6 \%\end{array}$ & $\begin{array}{l}\text { GAD-7 } \\
(\text { Anxiety } \geq 5 \text { ); } \\
\text { PHQ-9 } \\
\text { (Depression } \\
\geq 10 \text { ) }\end{array}$ & $\begin{array}{l}\text { Anxiety: } 38.2 \% \text {; } \\
\text { Depression } 10.5 \%\end{array}$ \\
\hline $\begin{array}{l}\text { Gupta AK et } \\
\text { al. (2020) }\end{array}$ & Nepal & $\begin{array}{l}\text { Cross- } \\
\text { sectional; } \\
\text { NR }\end{array}$ & $\begin{array}{l}\text { Online; } \\
\text { snowball } \\
\text { sampling }\end{array}$ & $\begin{array}{l}150 ; \text { healthcare } \\
\text { providers }\end{array}$ & 29.5 & 52.7 & $\begin{array}{l}\text { Graduate and } \\
\text { above } 52 \% ; \\
\text { nursing staff } 31.3 \\
\% \text {, faculty } \\
\text { members } 24.7 \%\end{array}$ & $\begin{array}{l}\text { GAD-7 } \\
(\text { Anxiety } \geq 5 \text { ); } \\
\text { PHQ-9 } \\
\text { (Depression } \\
\geq 10 \text { ) }\end{array}$ & $\begin{array}{l}\text { Anxiety: } 34 \% ; \\
\text { Depression: } 10 \%\end{array}$ \\
\hline $\begin{array}{l}\text { Gupta S. et } \\
\text { al. (2020)a }\end{array}$ & India & $\begin{array}{l}\text { Cross- } \\
\text { sectional; } \\
79.44 \%\end{array}$ & $\begin{array}{l}\text { Online; Quota } \\
\text { sampling }\end{array}$ & $\begin{array}{l}1124 ; \\
\text { healthcare } \\
\text { providers }\end{array}$ & $\begin{array}{l}\text { Mostly } \\
20-35 \\
\text { years }\end{array}$ & 36.1 & $\begin{array}{l}\text { Undergraduate } \\
\text { degree } 39.1 \% \text {, } \\
\text { graduate and } \\
\text { above } 59.5 \% \text {; } \\
\text { Doctors } 66.6 \% \text {, } \\
\text { Nurses } 18.4 \text {, } \\
\text { paramedics } 12 \% .\end{array}$ & $\operatorname{HADS}(>7)$ & $\begin{array}{l}\text { Anxiety: } 37.2 \% \text {; } \\
\text { Depression } 31.4 \%\end{array}$ \\
\hline $\begin{array}{l}\text { Gupta S. et } \\
\text { al. }(2020) b\end{array}$ & India & $\begin{array}{l}\text { Cross- } \\
\text { sectional; } \\
85.7 \%\end{array}$ & Online; NR & $\begin{array}{l}749 \text {; healthcare } \\
\text { providers }\end{array}$ & $\begin{array}{l}\text { Mostly } \\
20-35 \\
\text { years }\end{array}$ & 25.8 & $\begin{array}{l}\text { Undergraduate } \\
24.6 \% \text {, graduate } \\
\text { and above } 75.4 \% \text {; } \\
\text { Physicians } 100 \%\end{array}$ & $\operatorname{HADS}(>7)$ & $\begin{array}{l}\text { Anxiety: } 35.2 \% \text {; } \\
\text { Depression: } \\
28.2 \%\end{array}$ \\
\hline $\begin{array}{l}\text { Hasan et al. } \\
(2020)\end{array}$ & Pakistan & $\begin{array}{l}\text { Cross- } \\
\text { sectional; } \\
\text { NR }\end{array}$ & Online; NR & $\begin{array}{l}\text { 151; healthcare } \\
\text { providers }\end{array}$ & $\begin{array}{l}29 \\
( \pm 7.28)\end{array}$ & 56.3 & $\begin{array}{l}\text { Undergraduate } \\
\text { degree } 76.2 \% \\
\text { postgraduate } \\
\text { degree } 23.8 \% ; \\
\text { Physicians } 100 \%\end{array}$ & GAD-7 ( $\geq 5)$ & Anxiety: $63.58 \%$ \\
\hline $\begin{array}{l}\text { Hossain et } \\
\text { al. (2020) }\end{array}$ & Bangladesh & $\begin{array}{l}\text { Cross- } \\
\text { sectional; } \\
\text { NR }\end{array}$ & Online; NR & $\begin{array}{l}880 \text {; general } \\
\text { population }\end{array}$ & $\begin{array}{l}26.3 \\
( \pm 7.2)\end{array}$ & 30 & $\begin{array}{l}\text { Undergraduate } \\
34.1 \% \text {, graduate } \\
\text { and above } 34.8 \% \text {; } \\
\text { Students } 56 \% \text {, } \\
\text { Service holder } 31 \%\end{array}$ & GAD-7 $(\geq 10)$ & Anxiety: $49.1 \%$ \\
\hline
\end{tabular}




\begin{tabular}{|c|c|c|c|c|c|c|c|c|c|}
\hline $\begin{array}{l}\text { Imran et al. } \\
(2020) a\end{array}$ & Pakistan & $\begin{array}{l}\text { Cross- } \\
\text { sectional; } \\
\text { NR }\end{array}$ & Online; NR & $\begin{array}{l}337 \text {; healthcare } \\
\text { providers }\end{array}$ & $\begin{array}{l}30.4 \\
( \pm 6.7)\end{array}$ & 53 & $\begin{array}{l}\text { Physicians } 79 \% \text {, } \\
\text { nurses and } \\
\text { paramedical staff } \\
20 \%\end{array}$ & $\begin{array}{l}\text { GAD-7 } \\
(\text { Anxiety >7), } \\
\text { PHQ-9 } \\
\text { (Depression } \\
\geq 10 \text { ) }\end{array}$ & $\begin{array}{l}\text { Anxiety } 36.2 \% \text {; } \\
\text { Depression } 30 \%\end{array}$ \\
\hline $\begin{array}{l}\text { Imran et al. } \\
(2020) b\end{array}$ & Pakistan & $\begin{array}{l}\text { Cross- } \\
\text { sectional; } \\
88.6 \%\end{array}$ & Online; NR & $\begin{array}{l}\text { 10178; } \\
\text { healthcare } \\
\text { providers }\end{array}$ & 31.5 & 56.7 & $\begin{array}{l}\text { Postgraduate } \\
\text { trainees } 100 \%\end{array}$ & $\begin{array}{l}\text { GAD-7 } \\
(\text { Anxiety >5), } \\
\text { PHQ-9 } \\
\text { (Depression } \\
\geq 8 \text { ) }\end{array}$ & $\begin{array}{l}\text { Anxiety: 22.6\%; } \\
\text { Depression: } \\
26.4 \%\end{array}$ \\
\hline $\begin{array}{l}\text { Islam et al. } \\
(2020) a\end{array}$ & Bangladesh & $\begin{array}{l}\text { Cross- } \\
\text { sectional; } \\
\text { NR }\end{array}$ & $\begin{array}{l}\text { Online; } \\
\text { snowball } \\
\text { sampling }\end{array}$ & $\begin{array}{l}\text { 476; general } \\
\text { population }\end{array}$ & $\begin{array}{l}\text { Mostly } \\
21-24 \\
\text { years }\end{array}$ & 32.8 & $\begin{array}{l}\text { University student } \\
100 \%\end{array}$ & $\begin{array}{l}\text { GAD-7 } \\
\text { (Anxiety } \geq 5 \text { ), } \\
\text { PHQ-9 } \\
\text { (Depression } \\
\geq 5 \text { ) }\end{array}$ & $\begin{array}{l}\text { Anxiety: } 81.7 \% \text {; } \\
\text { Depression: } \\
82.4 \%\end{array}$ \\
\hline $\begin{array}{l}\text { Islam et al. } \\
(2020) b\end{array}$ & Bangladesh & $\begin{array}{l}\text { Cross- } \\
\text { sectional; } \\
97.4 \%\end{array}$ & Online; NR & $\begin{array}{l}\text { 1311; general } \\
\text { population }\end{array}$ & 23.54 & 39.6 & $\begin{array}{l}\text { Undergraduate } \\
\text { degree } 68.6 \% ; \\
\text { students } 81.6 \%\end{array}$ & GAD-7 $(\geq 10)$ & Anxiety: $37.3 \%$ \\
\hline $\begin{array}{l}\text { Jain et al. } \\
(2020)\end{array}$ & India & $\begin{array}{l}\text { Cross- } \\
\text { sectional; } \\
\text { NR }\end{array}$ & Online; NR & $\begin{array}{l}\text { 512; healthcare } \\
\text { providers }\end{array}$ & $\begin{array}{l}\text { Mostly } \\
<35 \\
\text { years }\end{array}$ & 44.3 & $\begin{array}{l}\text { Anesthesiology } \\
\text { residents } 68.4 \% \text {, } \\
\text { consultants } 31.6 \%\end{array}$ & GAD-7 $(\geq 5)$ & Anxiety: $74.2 \%$ \\
\hline $\begin{array}{l}\text { Khanal et } \\
\text { al. (2020) }\end{array}$ & Nepal & $\begin{array}{l}\text { Cross- } \\
\text { sectional; } \\
\text { NR }\end{array}$ & Online; NR & $\begin{array}{l}475 \text {; healthcare } \\
\text { providers }\end{array}$ & $\begin{array}{l}28.20 \\
( \pm 5.80)\end{array}$ & 52.6 & $\begin{array}{l}\text { Undergraduate } \\
58.3 \% \text {, graduate } \\
\text { and above } 21.9 \% \text {; } \\
\text { Nurses } 35.2 \text {, } \\
\text { Doctors } 33.9 \%\end{array}$ & $\operatorname{HADS}(>7)$ & $\begin{array}{l}\text { Anxiety: } 41.9 \% ; \\
\text { Depression } 37.5 \%\end{array}$ \\
\hline $\begin{array}{l}\text { Khanna et } \\
\text { al. (2020) }\end{array}$ & India & $\begin{array}{l}\text { Cross- } \\
\text { sectional; } \\
\text { NR }\end{array}$ & Online; NR & $\begin{array}{l}\text { 2355; } \\
\text { healthcare } \\
\text { providers }\end{array}$ & 42.5 & 43.3 & $\begin{array}{l}\text { Ophthalmology } \\
\text { resident } 15.2 \% \text {, } \\
\text { government } \\
\text { doctor } 12.8 \% \text {, } \\
\text { private } \\
\text { practitioner } 52.1 \% \text {, } \\
\text { Non-government } \\
\text { doctor } 19.9 \% \text {. }\end{array}$ & PHQ-9 $(\geq 5)$ & $\begin{array}{l}\text { Depression: } \\
32.6 \%\end{array}$ \\
\hline $\begin{array}{l}\text { Mamun et } \\
\text { al. (2020) }\end{array}$ & Bangladesh & $\begin{array}{l}\text { Cross- } \\
\text { sectional; } \\
91.52 \%\end{array}$ & Online; NR & $\begin{array}{l}\text { 10067; general } \\
\text { population }\end{array}$ & $\begin{array}{l}29.9 \\
( \pm 9.6)\end{array}$ & 43.9 & $\begin{array}{l}\text { Tertiary education } \\
80.8 \% ; 58.4 \% \\
\text { students, } 25.7 \% \\
\text { employed,3.6\% } \\
\text { unemployed }\end{array}$ & PHQ-9 $(\geq 10)$ & $\begin{array}{l}\text { Depression: } \\
33.3 \%\end{array}$ \\
\hline
\end{tabular}




\begin{tabular}{|c|c|c|c|c|c|c|c|c|c|}
\hline $\begin{array}{l}\text { Mani et al. } \\
(2020)\end{array}$ & India & $\begin{array}{l}\text { Cross- } \\
\text { sectional; } \\
\text { NR }\end{array}$ & $\begin{array}{l}\text { Online; } \\
\text { snowball } \\
\text { sampling }\end{array}$ & $\begin{array}{l}618 \text {; general } \\
\text { population }\end{array}$ & NR & 43 & $\begin{array}{l}\text { Graduate and } \\
\text { above } 67 \% \text {; student } \\
31 \% \text {, healthcare } \\
\text { worker } 20 \% \text {, } \\
\text { service holder } \\
21.9 \% \text {, } \\
\text { Businessperson } \\
3.5 \%\end{array}$ & GAD-7 ( $\geq 5)$ & Anxiety: $19.4 \%$ \\
\hline $\begin{array}{l}\text { Nisha S. et } \\
\text { al. (2020) }\end{array}$ & India & $\begin{array}{l}\text { Cross- } \\
\text { sectional; } \\
\text { NR }\end{array}$ & $\begin{array}{l}\text { N/R; } \\
\text { convenience } \\
\text { sampling }\end{array}$ & $\begin{array}{l}\text { 359; general } \\
\text { population }\end{array}$ & $\begin{array}{l}\text { Mostly } \\
18-21 \\
\text { years }\end{array}$ & 49.6 & $\begin{array}{l}\text { Medical Students } \\
100 \%\end{array}$ & $\begin{array}{l}\text { GAD-7 } \\
\text { (Anxiety } \geq 5 \text { ); } \\
\text { CES-D } \\
\text { (Depression } \\
\geq 5 \text { ) }\end{array}$ & $\begin{array}{l}\text { Anxiety: } 75.5 \% \text {; } \\
\text { Depression: } \\
74.6 \%\end{array}$ \\
\hline $\begin{array}{l}\text { Patabendig } \\
\text { e et al. } \\
(2020)\end{array}$ & Sri Lanka & $\begin{array}{l}\text { Cross- } \\
\text { sectional; } \\
\text { NR }\end{array}$ & $\begin{array}{l}\text { In-person; } \\
\text { quota } \\
\text { sampling }\end{array}$ & $\begin{array}{l}\text { 257; pregnant } \\
\text { participants }\end{array}$ & $\begin{array}{l}29.2 \\
( \pm 5.8)\end{array}$ & 100 & $\mathrm{~N} / \mathrm{R}$ & $\operatorname{HADS}(\geq 8)$ & $\begin{array}{l}\text { Anxiety } 17.5 \% \\
\text { Depression: } \\
19.5 \%\end{array}$ \\
\hline $\begin{array}{l}\text { Reddy et al. } \\
(2020)\end{array}$ & India & $\begin{array}{l}\text { Cross- } \\
\text { sectional; } \\
\text { NR }\end{array}$ & $\begin{array}{l}\text { Online; } \\
\text { respondent- } \\
\text { driven } \\
\text { sampling }\end{array}$ & $\begin{array}{l}\text { 891; general } \\
\text { population }\end{array}$ & $\begin{array}{l}\text { Mostly } \\
21-40 \\
\text { years }\end{array}$ & 47 & $\begin{array}{l}\text { Graduate and } \\
\text { above } 94 \% ; 34 \% \\
\text { students, } \\
\text { employed 53\%, } \\
\text { others } 8 \%\end{array}$ & DASS-21 & $\begin{array}{l}\text { Anxiety: } 15 \% ; \\
\text { Depression: } 22 \%\end{array}$ \\
\hline $\begin{array}{l}\text { Sandesh et } \\
\text { al. (2020) }\end{array}$ & Pakistan & $\begin{array}{l}\text { Cross- } \\
\text { sectional; } \\
\text { NR }\end{array}$ & Online; NR & $\begin{array}{l}\text { 112; healthcare } \\
\text { providers }\end{array}$ & $\mathrm{N} / \mathrm{R}$ & $\mathrm{N} / \mathrm{R}$ & $\mathrm{N} / \mathrm{R}$ & DASS-21 & $\begin{array}{l}\text { Anxiety: } 85.7 \% \text {; } \\
\text { Depression: } \\
72.3 \%\end{array}$ \\
\hline $\begin{array}{l}\text { Sebastian } \\
\text { et al. (2020) }\end{array}$ & India & $\begin{array}{l}\text { Cross- } \\
\text { sectional; } \\
\text { NR }\end{array}$ & online & $\begin{array}{l}1257 \text {; general } \\
\text { population }\end{array}$ & 29.3 & 54.4 & $\begin{array}{l}\text { Students } 39 \% \text {, } \\
\text { employed } 33.1 \% \text {, } \\
\text { unemployed } 13.1 \%\end{array}$ & $\begin{array}{l}\text { PHQ-4 } \\
\text { (Depression } \\
\geq 3 \text {, Anxiety } \\
\geq 9 \text { ) }\end{array}$ & $\begin{array}{l}\text { Anxiety: } 20 \% \text {, } \\
\text { Depression: } 18 \%\end{array}$ \\
\hline $\begin{array}{l}\text { Sharma et } \\
\text { al. (2020) }\end{array}$ & India & $\begin{array}{l}\text { Cross- } \\
\text { sectional; } \\
\text { NR }\end{array}$ & Landline & 62 ; Patients & 34.05 & 25.8 & $\begin{array}{l}\text { Student-33.9\%, } \\
\text { Businessman- } \\
\text { 17.7\%, Housewife- } \\
\text { 16.1\%, Daily wage } \\
\text { worker- } 12.9 \%\end{array}$ & CES-D $(\geq 10)$ & $\begin{array}{l}\text { Depression: } \\
62.9 \%\end{array}$ \\
\hline $\begin{array}{l}\text { Sheshtha et } \\
\text { al. (2020) }\end{array}$ & Nepal & $\begin{array}{l}\text { Cross- } \\
\text { sectional; } \\
\text { NR }\end{array}$ & $\begin{array}{l}\text { N/R; } \\
\text { convenience } \\
\text { sampling }\end{array}$ & $\begin{array}{l}\text { 101; healthcare } \\
\text { providers }\end{array}$ & $\mathrm{N} / \mathrm{R}$ & 57.4 & $\begin{array}{l}\text { Doctors 59.4\%, } \\
\text { Nurses } 40.6 \%\end{array}$ & GAD-7 & Anxiety: $73.3 \%$ \\
\hline $\begin{array}{l}\text { Sil et al. } \\
(2020)\end{array}$ & India & $\begin{array}{l}\text { Cross- } \\
\text { sectional; } \\
\text { NR }\end{array}$ & $\begin{array}{l}\text { Online; } \\
\text { snowball } \\
\text { sampling }\end{array}$ & $\begin{array}{l}41 \text {; healthcare } \\
\text { providers }\end{array}$ & $\begin{array}{l}\text { Mostly } \\
26-30 \\
\text { years }\end{array}$ & 41.5 & $\begin{array}{l}\text { Frontline } \\
\text { dermatologists } \\
\text { 100\%. } \\
\text { Postgraduate }\end{array}$ & PHQ-9 & $\begin{array}{l}\text { Depression: } \\
26.82 \%\end{array}$ \\
\hline
\end{tabular}




\begin{tabular}{|c|c|c|c|c|c|c|c|c|c|}
\hline & & & & & & & $\begin{array}{l}\text { trainee } 51.22 \% \\
\text { senior resident } \\
29.27 \% \text {, consultant } \\
19.51 \%\end{array}$ & & \\
\hline $\begin{array}{l}\text { Suryavansh } \\
\text { i et al. } \\
(2020)\end{array}$ & India & $\begin{array}{l}\text { Cross- } \\
\text { sectional; } \\
\text { NR }\end{array}$ & $\begin{array}{l}\text { Online; } \\
\text { snowball } \\
\text { sampling }\end{array}$ & $\begin{array}{l}\text { 197; healthcare } \\
\text { providers }\end{array}$ & $\begin{array}{l}\text { Mostly } \\
<30 \\
\text { years }\end{array}$ & 51.27 & $\begin{array}{l}24 \% \text { nurses, } 34 \% \\
\text { physicians, } 29 \% \\
\text { residents/interns } \\
\text { and } \\
13 \% \text { others. }\end{array}$ & $\begin{array}{l}\text { PHQ-9 } \\
\text { (Depression: } \\
\geq 5 \text { ), GAD } \\
\text { (Anxiety: } \geq 5 \text { ) }\end{array}$ & $\begin{array}{l}\text { Anxiety: 50\%; } \\
\text { Depression: } 47 \%\end{array}$ \\
\hline $\begin{array}{l}\text { Verma et } \\
\text { al. (2020) }\end{array}$ & India & $\begin{array}{l}\text { Cross- } \\
\text { sectional; } \\
93.9 \%\end{array}$ & $\begin{array}{l}\text { Online; } \\
\text { convenience } \\
\text { sampling }\end{array}$ & $\begin{array}{l}\text { 354; general } \\
\text { population }\end{array}$ & $\begin{array}{l}\text { Mostly } \\
18-25 \\
\text { years }\end{array}$ & 48.3 & $\begin{array}{l}\text { Graduate and } \\
\text { above } 91.8 \% \text {; } \\
\text { employed } 39.5 \% \text {, } \\
\text { unemployed } 60.5 \%\end{array}$ & $\begin{array}{l}\text { DASS-21 } \\
\text { (Anxiety >7, } \\
\text { Depression } \\
>9 \text { ) }\end{array}$ & $\begin{array}{l}\text { Anxiety: } 27.96 \% ; \\
\text { Depression: } \\
25.14 \%\end{array}$ \\
\hline $\begin{array}{l}\text { Wilson et } \\
\text { al. (2020) }\end{array}$ & India & $\begin{array}{l}\text { Cross- } \\
\text { sectional; } \\
\text { NR }\end{array}$ & Online; NR & $\begin{array}{l}350 \text {; healthcare } \\
\text { providers }\end{array}$ & 30.21 & 46.6 & $\begin{array}{l}84.3 \% \\
\text { doctors, } 15.7 \% \\
\text { nurses. }\end{array}$ & $\begin{array}{l}\text { GAD-7 } \\
\text { (Anxiety } \geq 5, \\
\text { PHQ-9 } \\
\text { (Depression } \\
\geq 10 \text { ) }\end{array}$ & $\begin{array}{l}\text { Anxiety: } 66.29 \% \\
\text { Depression: } \\
49.43 \%\end{array}$ \\
\hline $\begin{array}{l}\text { Zubayer et } \\
\text { al. (2020) }\end{array}$ & Bangladesh & $\begin{array}{l}\text { Cross- } \\
\text { sectional; } \\
\text { NR }\end{array}$ & Online; NR & $\begin{array}{l}\text { 1146; general } \\
\text { population }\end{array}$ & 26.38 & 40.8 & $\begin{array}{l}\text { Graduate and } \\
\text { above } 78.5 \% \text {; } \\
\text { student } 58.6 \% \text {, } \\
\text { housewife } 3.9 \% \text {, } \\
\text { govt./private } \\
\text { employee } \\
\text { 16.6\%, } \\
\text { doctor/health } \\
\text { worker } 12.7 \% \text {, and } \\
\text { unemployed } 8.2 \% \text {. }\end{array}$ & $\begin{array}{l}\text { DASS- } 21 \\
\text { (Anxiety } \geq 10, \\
\text { Depression } \\
\geq 14 \text { ) }\end{array}$ & $\begin{array}{l}\text { Anxiety } 46 \% ; \\
\text { Depression } 47.2 \%\end{array}$ \\
\hline
\end{tabular}

Note: NR: Not reported, GAD-7: Generalized Anxiety Disorder 7-item, DASS-21: Depression, Anxiety and Stress Scale - 21 Items, HADS: Hospital Anxiety and Depression Scale, PHQ-4:

Patient Health Questionnaire-4, PHQ-9: Patient Health Questionnaire (PHQ)-9, SRQ-20: Self Reporting Questionnaire 20-items, CES-D: Center for Epidemiologic Studies Depression Scale 
In the Newcastle-Ottawa Quality Assessment, 24 studies were identified to have a high risk of bias, whereas 11 studies had a low risk of bias with a quality score of 3 and above (Supplementary file A). The prevalence rates of anxiety and depression were calculated using separate metaanalyses, including meta-regression, sub-group estimates, sensitivity analyses, and assessments of publication bias.

\subsection{Prevalence of anxiety during COVID-19 in South Asia}

\subsubsection{Pooled prevalence of anxiety}

A total of 31 studies reported the prevalence of anxiety during COVID-19 in different samples from South Asian countries ${ }^{46-56,58,59,61-66,88,69,71-80}$. These studies used different scales to measure anxiety in respective samples, including the Generalized Anxiety Disorder 7-item (GAD-7) scale $(n=16)$, Depression, Anxiety and Stress Scale - 21 Items (DASS-21) scales $(n=9)$, Hospital Anxiety and Depression Scale (HADS) ( $n=4)$, Patient Health Questionnaire-4 (PHQ-4) $(n=1)$, and Self Reporting Questionnaire 20-items (SRQ-20) scale $(n=1)$. These scales were used with different cut-off values to determine the overall prevalence as well as the severity of anxiety. In randomeffects model, the pooled prevalence of anxiety was $41.3 \%$ (95\% CI: $34.7-48.1, I^{2}=99.18 \%$ ) among 28877 participants in 31 studies (Figure 2).

\subsubsection{Meta-regression and subgroup analyses}

The pooled prevalence of anxiety was significantly associated with the percentage of female participants in respective studies $(p=.04)$ (Supplementary file B). Moreover, there was no correlation between the pooled prevalence and mean age of the participants $(p=.98)$ or the quality score of the studies $(p=.47)$. The subgroup analyses revealed different prevalence estimates in pooled samples (Table 3). The pooled prevalence of anxiety was higher $(46.49 \%, 95 \%$ CI: $\left.36.99-56.6, I^{2}=98.5 \%\right)$ among female participants compared to the male participants (41.13\%, 95\% CI: 32.99-49.51, $\left.I^{2}=98.6 \%\right)$.

Among the South Asian countries reporting the prevalence of anxiety, Bangladesh had the highest prevalence ( $52.3 \%, 95 \%$ CI: $\left.41-63.6, I^{2}=98.67 \%\right)$ followed by Pakistan $(50.4 \%, 95 \%$ CI: 30.570.2, $\left.I^{2}=99 \%\right)$, Nepal (49.6\%, 95\% CI: 30.6-68.7, $\left.I^{2}=95.45 \%\right)$, and India (34.7\%, 95\% CI: $25.4-44.7, I^{2}$ 
=99.13\%). Moreover, the pooled prevalence of anxiety among the healthcare providers was $43.6 \%$ (95\% CI: 33.1-54.5, $I^{2}=99.15 \%$ ), whereas general population had a prevalence of $40.7 \%$ ( $95 \%$ CI: $\left.31.6-50.1, I^{2}=99.15\right)$.

Furthermore, the prevalence of anxiety varied across samples that were assessed using different scales. The prevalence of anxiety was higher (49.2\%, 95\% CI: $\left.39.1-59.3, I^{2}=99.34 \%\right)$ in GAD-7 scale compared to samples that were assessed by DASS-21 (34.2\%, 95\% CI: $\left.19.2-51, I^{2}=99.35 \%\right)$ and HADS (32.8\%, 95\% CI: 25.1-41, $\left.I^{2}=94.39 \%\right)$ scales. Also, studies with high risk of bias according to the Newcastle-Ottawa Quality Assessment has a higher prevalence (43.9\%, 95\% CI: 36.4-51.6, $I^{2}=$ 99.19\%) compared to studies with low risk of bias (36\%, 95\% CI: 20.4-53.2, $\left.I^{2}=99.26 \%\right)$.

\begin{tabular}{|c|c|c|c|}
\hline Studies & Estimate $195 \%$ & C.I.) & $\mathrm{Ev} / \mathrm{Tr} t$ \\
\hline Ahmad et al. 2020 & $0.253 \quad(0.211$ & 0.297 ) & $99 / 392$ \\
\hline Ahmed et al. 2020 & $0.398 \quad(0.355$ & $0.441)$ & $199 / 500$ \\
\hline Amin et al. 2020 & $0.429 \quad 0.380$ & $0.479)$ & $167 / 389$ \\
\hline Banna et al. 2020 & $0.579 \quad(0.553$ & $0.604)$ & $826 / 1427$ \\
\hline Chatterjee et al. 2020 & $0.395 \quad 0.318$ & $0.474)$ & $60 / 152$ \\
\hline Chew et al. a 2020 & $0.008 \quad(0.001$ & $0.020)$ & $3 / 384$ \\
\hline Chew et al. b 2020 & $0.171 \quad 0.137$ & $0.209)$ & $73 / 426$ \\
\hline Desai et al. 2020 & $0.415 \quad(0.391$ & $0.440)$ & $638 / 1537$ \\
\hline Grover et al. 2020 & $0.382 \quad(0.359$ & $0.406)$ & $644 / 1685$ \\
\hline Gupta AK et al. 2020 & $0.340 \quad(0.266$ & $0.418)$ & $51 / 150$ \\
\hline Gupta et al. a 2020 & $0.372(0.344$ & $0.400)$ & $418 / 1124$ \\
\hline Gupta et al. b 2020 & $0.352 \quad(0.319$ & $0.387)$ & $264 / 749$ \\
\hline Hasan et al. 2020 & $0.636 \quad 0.557$ & $0.711)$ & $96 / 151$ \\
\hline Hossain et al. 2020 & $0.491 \quad 0.458$ & $0.524)$ & $432 / 880$ \\
\hline Imran et al. a 2020 & $0.362 \quad 0.311$ & $0.414)$ & $122 / 337$ \\
\hline Imran et al. b 2020 & $0.226(0.218$ & $0.234)$ & $2301 / 10178$ \\
\hline Islam et al. a 2020 & $0.817 \quad(0.781$ & $0.851)$ & $389 / 476$ \\
\hline Islam et al. b 2020 & $0.373 \quad 0.347$ & $0.399)$ & $489 / 1311$ \\
\hline Jain et al. 2020 & $0.742(0.703$ & $0.779)$ & $380 / 512$ \\
\hline Khanal et al. 2020 & $0.419 \quad(0.375$ & $0.464)$ & $199 / 475$ \\
\hline Mani et al. 2020 & $0.194 \quad 0.164$ & $0.226)$ & $120 / 618$ \\
\hline Nisha S et al. 2020 & $0.755 \quad(0.709$ & $0.798)$ & $271 / 359$ \\
\hline Patabendige et al. 2020 & $0.175 \quad(0.131$ & $0.224)$ & $45 / 257$ \\
\hline Reddy et al. 2020 & $0.155(0.132$ & $0.179)$ & $138 / 891$ \\
\hline Sandesh et al. 2020 & $0.857(0.786$ & $0.916)$ & $96 / 112$ \\
\hline Sebastian et al. 2020 & $0.200 \quad(0.179$ & $0.223)$ & $252 / 1257$ \\
\hline Sheshtha et al. 2020 & $0.733(0.642$ & $0.815)$ & $74 / 101$ \\
\hline Suryavanshi et al. 2020 & $0.497(0.428$ & $0.567)$ & $98 / 197$ \\
\hline Verma et al. 2020 & $0.280 \quad(0.234$ & $0.328)$ & $99 / 354$ \\
\hline Wilson et al. 2020 & $0.663(0.612$ & $0.712)$ & $232 / 350$ \\
\hline Zubayer et al. 2020 & $0.460 \quad(0.431$ & $0.489)$ & $527 / 1146$ \\
\hline Overall $\left(I^{\wedge} 2=99.18 \%, P<0.001\right)$ & $0.413 \quad(0.347$ & $0.481)$ & $9802 / 28877$ \\
\hline
\end{tabular}

Figure 2: Forest plot of the pooled prevalence of anxiety

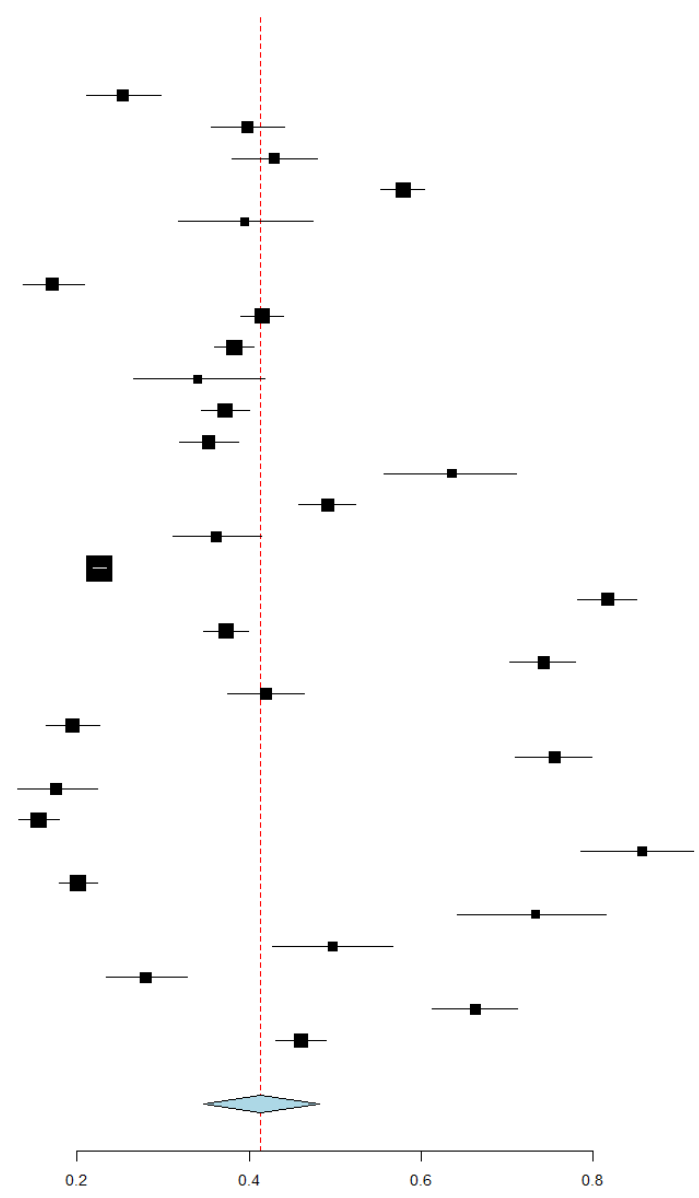


The prevalence of anxiety varied across subgroups based on the severity of symptoms among the participants. The prevalence was highest for mild anxiety $\left(27.24 \%, 95 \%\right.$ CI: $19.38-35.89, I^{2}=$ 99.3\%) followed by moderate (14.68\%, 95\% CI: $\left.12.3-17.22, I^{2}=94.7 \%\right)$ and severe anxiety $(9.94 \%$, 95\% CI: 7.09-13.2, $\left.I^{2}=97.6 \%\right)$.

Studies
Overall
- Ahmad et al. (2020)
- Ahmed et al. (2020)
- Amin et al. (2020)
- Banna et al. (2020)
- Chatterjee et al. (2020)
- Chew et al. a (2020)
- Chew et al. (2020)b
- Desai et al. (2020)
- Grover et al. (2020)
- Gupta AK et al. (2020)
- Gupta et al. (2020)a
- Gupta et al. (2020)b
- Hasan et al. (2020)
- Hossain et al. (2020)
- Imran et al. (2020)a
- Imran et al. (2020)b
- Islam et al. (2020)a
- Islam et al. (2020)b
- Jain et al. (2020)
- Khanal et al. (2020)
- Mani et al. (2020)
- Nisha S et al. (2020)
- Patabendige et al. (2020)
- Reddy et al. (2020)
- Sandesh et al. (2020)
- Sebastian et al. (2020)
- Sheshtha et al. (2020)
- Suryavanshi et al. (2020)
- Verma et al. (2020)
- Wilson et al. (2020)
- Zubayer et al. (2020)

$\begin{array}{lll}\text { Estimate }(95 \% & C . I .) \\ 0.413 & (0.347, & 0.481) \\ & & \\ 0.419 & (0.351, & 0.489) \\ 0.414 & (0.346, & 0.484) \\ 0.413 & (0.345, & 0.482) \\ 0.408 & (0.342, & 0.475) \\ 0.414 & (0.346, & 0.483) \\ 0.433 & (0.368, & 0.499) \\ 0.422 & (0.354, & 0.492) \\ 0.413 & (0.344, & 0.484) \\ 0.414 & (0.344, & 0.486) \\ 0.416 & (0.348, & 0.485) \\ 0.415 & (0.345, & 0.486) \\ 0.415 & (0.347, & 0.486) \\ 0.406 & (0.339, & 0.475) \\ 0.411 & (0.343, & 0.480) \\ 0.415 & (0.347, & 0.485) \\ 0.420 & (0.350, & 0.491) \\ 0.399 & (0.336, & 0.464) \\ 0.415 & (0.345, & 0.486) \\ 0.402 & (0.337, & 0.468) \\ 0.413 & (0.345, & 0.483) \\ 0.421 & (0.353, & 0.491) \\ 0.402 & (0.336, & 0.468) \\ 0.422 & (0.354, & 0.491) \\ 0.423 & (0.355, & 0.492) \\ 0.398 & (0.332, & 0.466) \\ 0.421 & (0.352, & 0.491) \\ 0.403 & (0.336, & 0.471) \\ 0.411 & (0.343, & 0.480) \\ 0.418 & (0.350, & 0.488) \\ 0.405 & (0.339, & 0.473) \\ 0.412 & (0.343, & 0.482) \\ & & \end{array}$

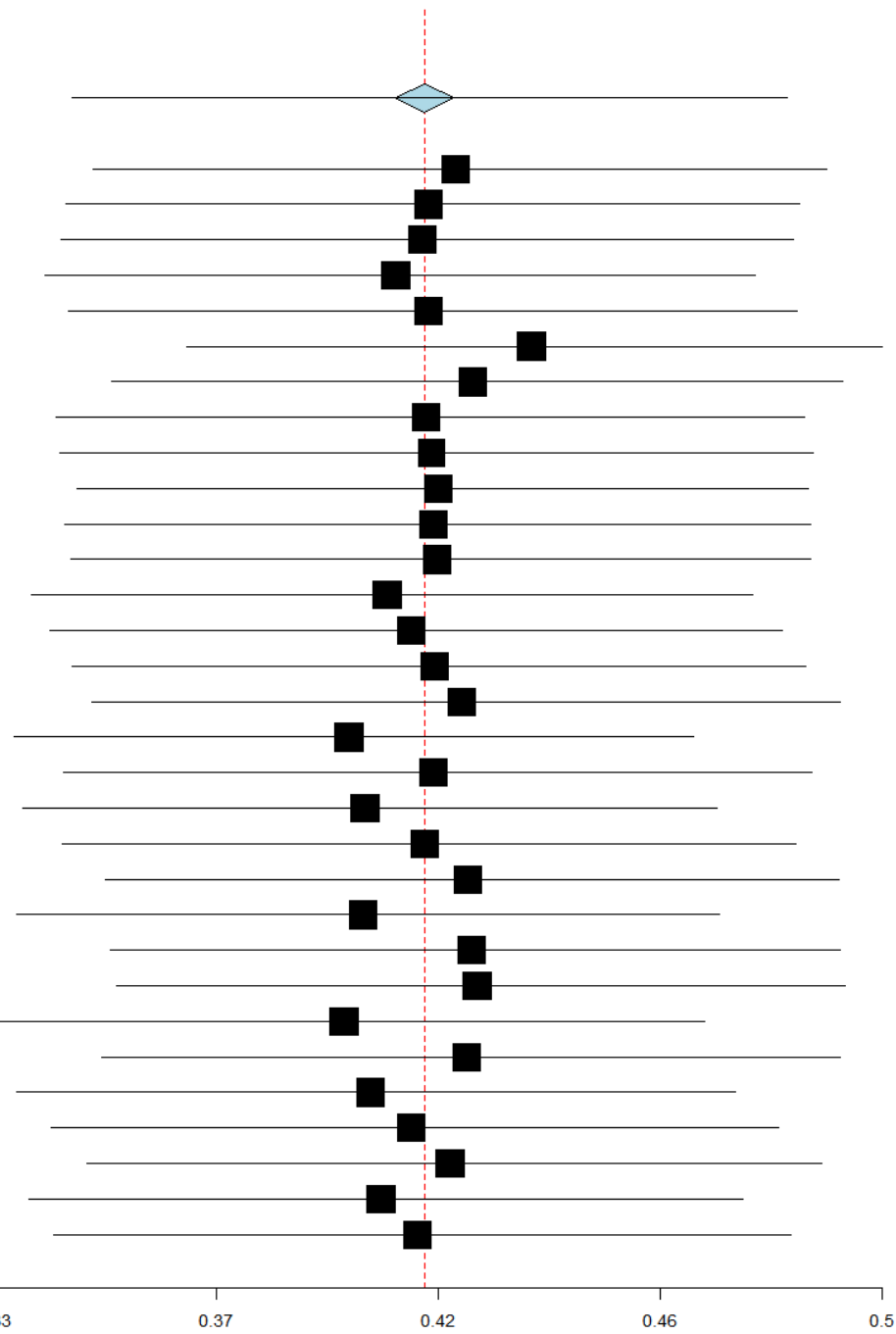

Figure 3: Sensitivity analysis (leave one out) for studies reporting anxiety prevalence 


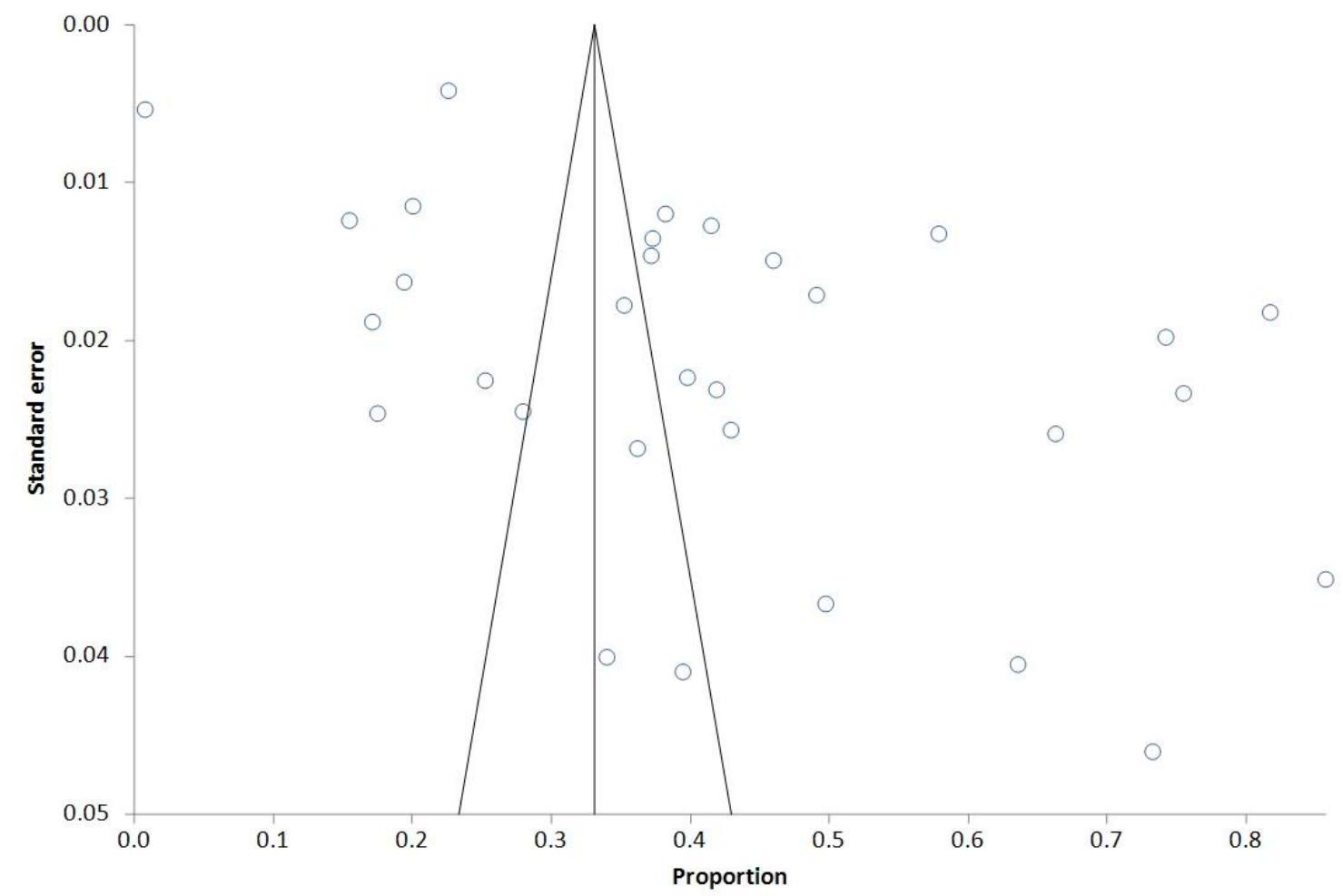

Figure 4: Funnel plot of the publication bias in studies reporting anxiety prevalence

\subsubsection{Sensitivity analysis and publication bias}

In the sensitivity analysis, the lowest prevalence (39.8\%, 95\% CI: 33.2-46.6) was observed after excluding the study by Sandesh and colleagues ${ }^{65}$, whereas the highest prevalence $(43.3 \%, 95 \% \mathrm{CI}$ : 36.8-49.9) was found after excluding a study by Chew and colleagues. The exclusion of each study one-by-one from the meta-analytic model did not change the pooled prevalence of anxiety substantially (Figure 3). The visual inspection of the funnel plot revealed high publication bias (Figure 4 ), which was confirmed by Egger's test $(p=.0002)$. 


\subsection{Prevalence of depression during COVID-19 in South Asia}

\subsubsection{Pooled prevalence of depression}

Twenty-eight studies reported the prevalence of depression during COVID-19 in different samples from South Asian countries ${ }^{47-49,52-54,57-60,62-67,69-80}$. The Patient Health Questionnaire (PHQ)9 was the most frequently used instrument $(n=11)$, followed by Depression, Anxiety and Stress Scale - 21 Items (DASS-21) scales ( $n=9)$, Hospital Anxiety and Depression Scale (HADS) $(n=4)$, Center for Epidemiologic Studies Depression Scale (CES-D) $(n=2)$, Patient Health Questionnaire4 (PHQ-4) $(n=1)$, and Self Reporting Questionnaire 20-items (SRQ-20) scale $(n=1)$. These scales had different constructs measuring overall depression and the severity of the symptoms using multiple cut-off values. The pooled prevalence of depression was 34.1\% (95\% CI: 28.9-39.4, $I^{2}=$ 99\%) among 37437 participants in 28 studies (Figure 5).

\footnotetext{
Studies

Ahmed et al. 2020

Amin et al. 2020

Banna et al. 2020

Chatterjee et al. 2020

Chew et al. a 2020

Chew et al. b 2020

Desai et al. 2020

Grover et al. 2020

Gupta AK et al. a 2020

Gupta et al. a 2020

Gupta et al. b 2020

Imran et al. a 2020

Imran et al. b 2020

Islam et al. a 2020

Khanal et al. 2020

Khanna et al. 2020

Mamun et al. 2020

Nisha S et al. 2020

Patabendige et al. 2020

Reddy et al. 2020

Sandesh et al. 2020

Sebastian et al. 2020

Sharma et al. 2020

Sil et al. 2020

Suryavanshi et al. 2020

Verma et al. 2020

Wilson et al. 2020

Zubayer et al. 2020

Estimate (95응 C.I.)

$0.430(0.387,0.474)$

$0.429(0.380,0.479)$

$0.337(0.313,0.362)$

$0.349(0.275,0.426)$

$0.008(0.001,0.020)$

$0.124(0.095,0.158)$

$0.470(0.445,0.495)$

$0.105(0.091,0.120)$

$0.080(0.041,0.129)$

$0.315(0.288,0.342)$

$0.282(0.250,0.314)$

$0.279(0.232,0.328)$

$0.264(0.255,0.272)$

$0.824(0.788,0.857)$

$0.375(0.332,0.419)$

$0.326(0.307,0.345)$

$0.333(0.323,0.342)$

$0.747(0.700,0.790)$

$0.195(0.148,0.245)$

$0.224(0.198,0.252)$

$0.723(0.636,0.803)$

$0.180(0.159,0.202)$

$0.629(0.505,0.746)$

$0.268(0.142,0.416)$

$0.467(0.398,0.537)$

$0.251(0.208,0.298)$

$0.494(0.442,0.547)$

$0.472(0.443,0.501)$

Overall $\left(I^{\wedge} 2=99 \%, P<0.001\right) \quad 0.341 \quad(0.289,0.394) \quad 11684 / 37437$

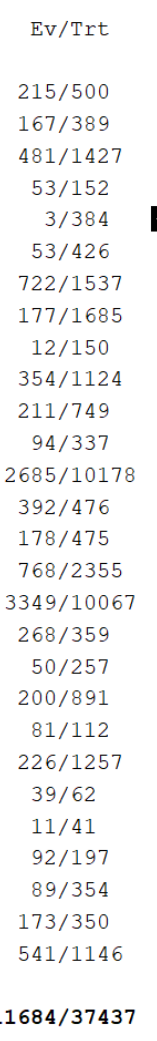

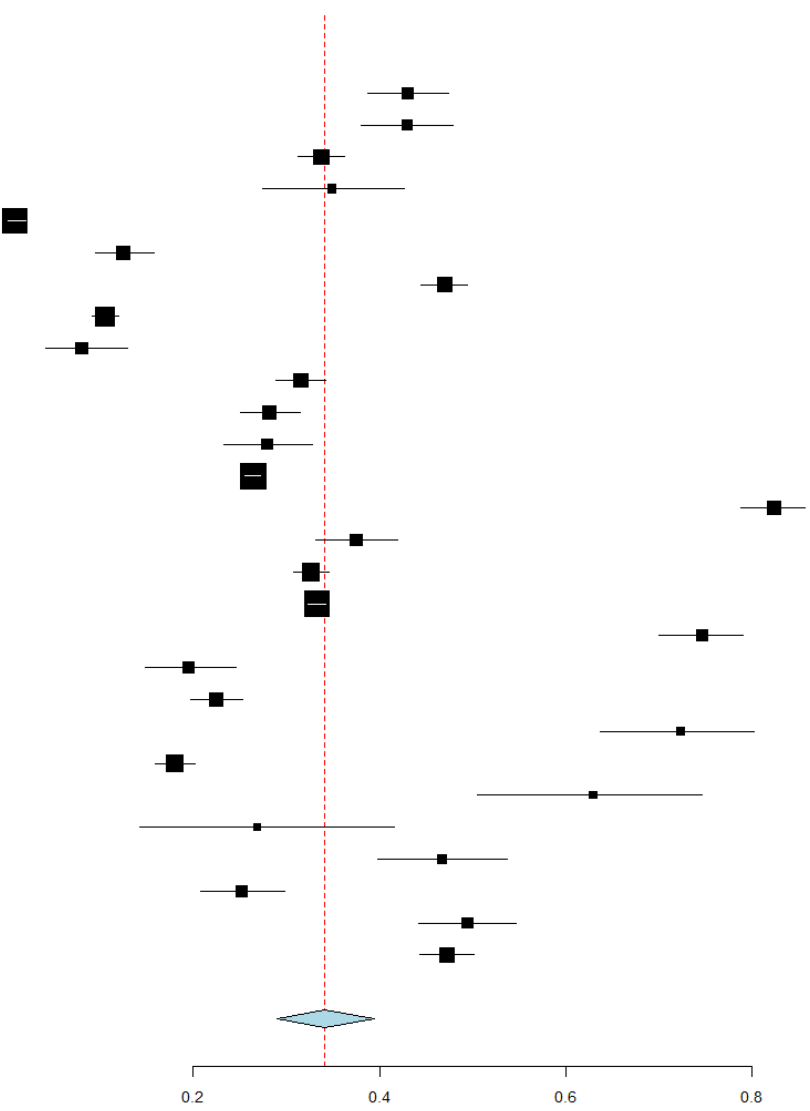

Figure 5: Forest plot of the pooled prevalence of depression 


\subsubsection{Meta-regression and subgroup analyses}

In meta-regression analyses, the prevalence of depression was not significantly associated with the mean age of the participants $(p=.784)$ and the quality scores of the studies $(p=.48)$ (Supplementary file C). However, the percentage of female participants was significantly associated $(p=.03)$ with the prevalence of depression. Moreover, gender was not a significant moderator according to the subgroup analyses (Table 3). The prevalence was similar among male (36.7\%, 95\% CI: 29.97-43.6, $\left.I^{2}=98.6 \%\right)$ and female (37.8\%, 95\% CI: 31.7-44.1, $\left.I^{2}=97.8 \%\right)$ participants during COVID-19.

At the country level, Bangladesh had the highest prevalence of depression (48.2\%, 95\% CI: 34.861.8, $\left.I^{2}=99.3 \%\right)$, followed by Pakistan (41.6\%, 95\% CI: 27.5-56.4, $\left.I^{2}=97.92 \%\right)$, India (30.7\%, 95\% CI: 22.3-39.8, $\left.I^{2}=99.05 \%\right)$, and Nepal (20.9\%, 95\% CI: 1.2-55.3, $\left.I^{2}=98.42 \%\right)$. The prevalence of depression was higher in general population (39\%, 95\% CI: $\left.29-49.5, I^{2}=99.44 \%\right)$ compared to the healthcare providers (29.9\%, 95\% CI: 23.9-36.2, $\left.I^{2}=98.12 \%\right)$.

The prevalence of depression varied across commonly used scales. For example, studies with GAD-7 had a higher prevalence (34.7\%, 95\% CI: 27.1-42.7, $\left.I^{2}=99.31 \%\right)$, whereas studies with DASS21 and HADS had similar prevalence (29.8\%, 95\% CI: $18.3-42.7, I^{2}=98.95 \%$ vs $29.2 \%, 95 \%$ CI: $23.6-$ $\left.35.1, I^{2}=89.89 \%\right)$. Moreover, studies with a high risk of bias had a higher pooled prevalence $\left(38.5 \%, 95 \%\right.$ CI: $\left.31.6-45.7, I^{2}=99.05 \%\right)$ compared to studies with a low risk of bias $(23.8 \%, 95 \%$ CI: $\left.14.6-34.5, I^{2}=99 \%\right)$.

The pooled prevalence was calculated for subgroups based on the severity of depressive symptoms. The prevalence of mild depression was 25.11\% (95\% CI: 17.72-33.29, $I^{2}=99.3 \%$ ), whereas the prevalence of moderate and severe depression was 13.91\% (95\% CI: 11.13-16.95, $I^{2}=$ 96.6\%) and $11.97 \%$ (95\% CI: 8.29-16.2, $\left.I^{2}=98.5 \%\right)$. 
Table 3: Subgroup analyses of the prevalence of anxiety and depression

\begin{tabular}{|c|c|c|c|}
\hline Groups & Subgroups & Anxiety & Depression \\
\hline \multirow[t]{4}{*}{ Gender } & \multirow[t]{2}{*}{ Female } & 46.49\%, 95\% CI: 36.55-56.6 & $37.8 \%, 96 \%$ CI: $31.7-44.1$ \\
\hline & & $I^{2}=98.5 \%$ & $I^{2}=97.8 \%$ \\
\hline & \multirow[t]{2}{*}{ Male } & 41.13\%, 95\% CI: 32.99-49.51 & $36.7 \%, 95 \%$ CI: $29.97-43.6$ \\
\hline & & $I^{2}=98.6 \%$ & $I^{2}=98.6 \%$ \\
\hline \multirow[t]{8}{*}{ Country } & \multirow[t]{2}{*}{ Bangladesh } & $52.3 \%, 95 \%$ CI: $41-63.6$ & $48.2 \%, 95 \%$ CI: $34.8-61.8$ \\
\hline & & $I^{2}=98.67 \%$ & $I^{2}=99.3 \%$ \\
\hline & \multirow[t]{2}{*}{ India } & $34.7 \%, 95 \%$ CI: $25.4-44.7$ & $30.7 \%, 95 \%$ CI: 22.3-39.8 \\
\hline & & $I^{2}=99.13 \%$ & $I^{2}=99.05 \%$ \\
\hline & \multirow[t]{2}{*}{ Nepal } & $49.6 \%, 95 \%$ CI: 30.6-68.7 & 20.9\%, 95\% CI: $1.2-55.3$ \\
\hline & & $I^{2}=95.45 \%$ & $I^{2}=98.42 \%$ \\
\hline & \multirow[t]{2}{*}{ Pakistan } & $50.4 \%, 95 \%$ CI: $30.5-70.2$ & $41.6 \%, 95 \%$ CI: 27.5-56.4 \\
\hline & & $I^{2}=99 \%$ & $I^{2}=97.92 \%$ \\
\hline \multirow{4}{*}{$\begin{array}{l}\text { Population } \\
\text { Groups }\end{array}$} & \multirow[t]{2}{*}{ General population } & $40.7 \%, 95 \%$ CI: $31.6-50.1$ & $39 \%, 95 \%$ CI: $29-49.5$ \\
\hline & & $I^{2}=99.15 \%$ & $I^{2}=99.44 \%$ \\
\hline & \multirow{2}{*}{$\begin{array}{l}\text { Healthcare } \\
\text { providers }\end{array}$} & $43.6 \%, 95 \%$ CI: $33.1-54.5$ & 29.9\%, 95\% CI: 23.9-36.2 \\
\hline & & $I^{2}=99.15 \%$ & $I^{2}=98.12 \%$ \\
\hline \multirow[t]{6}{*}{ Scales } & \multirow{2}{*}{$\begin{array}{l}\text { GAD-7 (Anxiety), } \\
\text { PHQ-9 (Depression) }\end{array}$} & $49.2 \%, 95 \%$ CI: 39.1-59.3 & 34.7\%, 95\% CI: 27.1-42.7 \\
\hline & & $I^{2}=99.34 \%$ & $I^{2}=99.31 \%$ \\
\hline & \multirow[t]{2}{*}{ DASS-21 } & $34.2 \%, 95 \%$ CI: $19.2-51$ & $29.8 \%, 95 \%$ CI: $18.3-42.7$ \\
\hline & & $I^{2}=99.35 \%$ & $I^{2}=98.95 \%$ \\
\hline & \multirow[t]{2}{*}{ HADS } & $32.8 \%, 95 \%$ CI: $25.1-41$ & 29.2\%, 95\% CI: 23.6-35.1 \\
\hline & & $I^{2}=94.39 \%$ & $I^{2}=89.89 \%$ \\
\hline \multirow[t]{4}{*}{ Risk of bias } & \multirow{2}{*}{$\begin{array}{l}\text { Studies with low } \\
\text { risk of bias }\end{array}$} & 36\%, 95\% CI: 20.4-53.2 & $23.8 \%, 95 \%$ CI: $14.6-34.5$ \\
\hline & & $I^{2}=99.26 \%$ & $I^{2}=99 \%$ \\
\hline & \multirow[t]{2}{*}{$\begin{array}{l}\text { Studies with high } \\
\text { risk of bias }\end{array}$} & $43.9 \%, 95 \%$ CI: $36.4-51.6$ & $38.5 \%, 95 \%$ CI: $31.6-45.7$ \\
\hline & & $I^{2}=99.19 \%$ & $I^{2}=99.05 \%$ \\
\hline \multirow[t]{6}{*}{ Severity } & \multirow[t]{2}{*}{ Mild } & 27.24\%, 95\% CI: $19.38-35.89$ & $\begin{array}{l}25.11 \%, 95 \% \text { CI: } 17.72- \\
33.29\end{array}$ \\
\hline & & $I^{2}=99.3 \%$ & $I^{2}=99.3 \%$ \\
\hline & \multirow[t]{2}{*}{ Moderate } & 14.68\%, 95\% CI: $12.3-17.22$ & $\begin{array}{l}\text { 13.91\%, 95\% CI: } 11.13- \\
16.95\end{array}$ \\
\hline & & $I^{2}=94.7 \%$ & $I^{2}=96.6 \%$ \\
\hline & \multirow[t]{2}{*}{ Severe } & 9.94\%, 95\% CI: 7.09-13.2 & 11.97\%, 95\% CI: 8.29-16.2 \\
\hline & & $I^{2}=97.6 \%$ & $I^{2}=98.5 \%$ \\
\hline
\end{tabular}




\subsubsection{Sensitivity analysis and publication bias}

We excluded each study one-by-one from the meta-analytic model in the sensitivity analysis. The findings showed no significant changes in the pooled prevalence of depression (Figure 6). The lowest prevalence was observed after excluding a study by Islam and colleagues (32.2\%, 95\% CI: 27.6-37 $)^{54}$, whereas the highest prevalence was found after excluding a study by Chew and colleagues $(35.9 \%, 95 \% \mathrm{CI}: 31-41.1)^{77}$. Furthermore, a high publication bias was observed in the visual assessment of the funnel plot (Figure 7), which was validated by Egger's test results, indicating a significant publication bias $(p=.05)$.

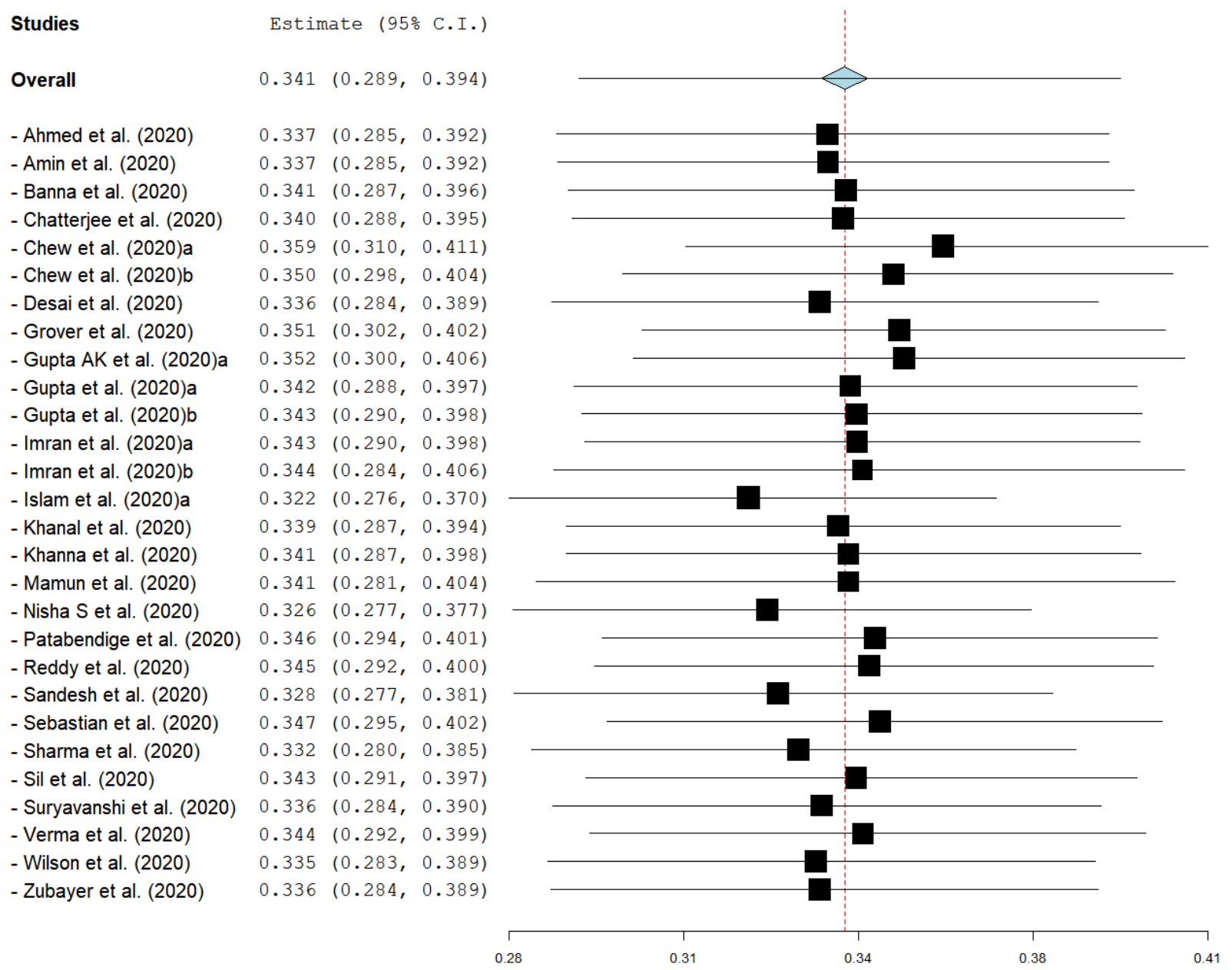

Figure 6: Sensitivity analysis (leave one out) for studies reporting depression prevalence 


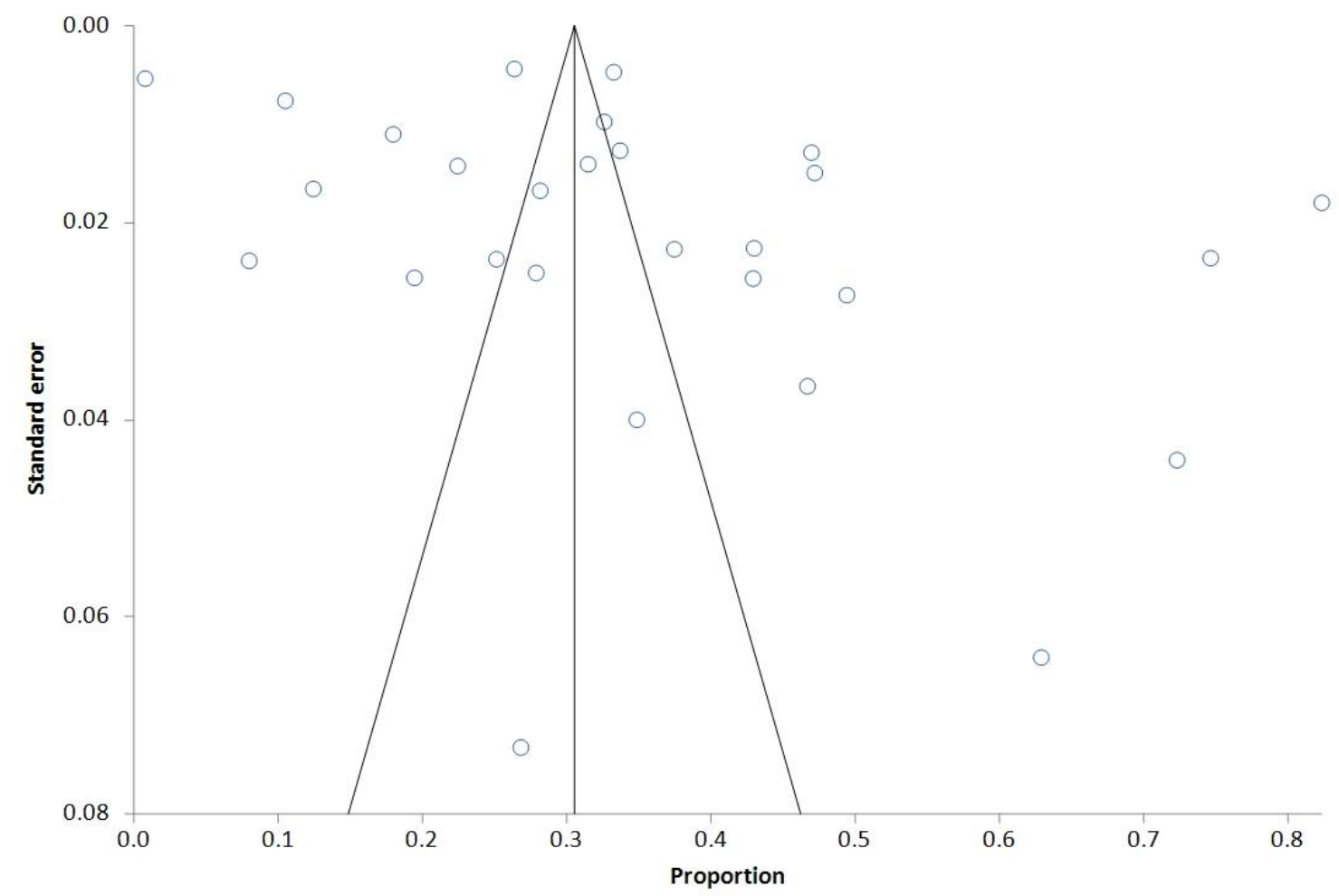

Figure 7: Funnel plot of the publication bias in studies reporting depression prevalence

\section{Discussion}

\subsection{Overview and interpretation of the synthesized findings}

To the best of our knowledge, this is the first quantitative synthesis of the epidemiological burden of anxiety and depression during the COVID-19 pandemic in South Asian region with country-level estimates. We systematically identified 35 cross-sectional studies and quantitatively evaluated the pooled prevalence in the collective sample alongside estimating the prevalence rates in different sub-groups and populations in this region. The overall prevalence of anxiety and depression was $41.3 \%$ and $34.1 \%$, respectively. Moreover, women had a higher prevalence of both disorders (anxiety 46.49\%, depression 37.8\%) compared to men (anxiety $41.13 \%$, depression $36.7 \%$ ). The percentage of female percentage was significantly associated with the prevalence of anxiety and depression in this review. 
Bangladesh (anxiety 52.3\%, depression 48.2\%) and Pakistan (anxiety 50.4\%, depression 41.6\%) had higher prevalence rates compared to other South Asian countries. Furthermore, the prevalence estimates varied among the healthcare providers and general population, studies with different measurement instruments, and subgroups with varying severity of anxiety and depression. Specifically, the prevalence of severe symptoms was critically high (anxiety 9.94\%, depression 11.97\%) among samples included in this review. The $I^{2}$ statistics of the pooled estimates ranged from $89.89 \%$ to $99.44 \%$, indicating high heterogeneity and inconsistency among studies included in this review. Also, meta-analytic models for anxiety and depression indicated high publication bias. These findings, alongside a high prevalence of those disorders during this pandemic, require a critical assessment of the evidence from multiple perspectives.

During the early months of COVID-19 pandemic, the scientific literature on the psychosocial challenges associated with pandemic was limited compared to the infectious diseases literature ${ }^{81}$. Moreover, a major proportion of the literature was published from China and many western countries that were initially affected by this pandemic ${ }^{9,40,81}$. Compared to those regions, studies from South Asia as well as the global south has been limited in number and may not have explored how this pandemic has impacted psychosocial wellbeing in these populations ${ }^{7-9}$. Therefore, the findings of the available literature may not reflect the accurate burden of anxiety and depression during this pandemic in the South Asian population.

Another perspective on evidence-based mental health may inform that this review only emphasized on cross-sectional evidence that may not inform the psychological impacts that can be attributable to this pandemic alone. As there is a scarcity of cohort studies focusing on mental health problems in South Asia, the available syntheses of previous research reported in epidemiological reviews can offer meaningful insights. An umbrella review of reviews identified 15 reviews that reported a high burden of anxiety and depression in South Asian countries ${ }^{37}$. Moreover, a meta-analytic review found that the prevalence of anxiety, depressive symptoms were $25.8 \%$ and $26.8 \%$, respectively ${ }^{36}$. These syntheses of the previous research highlight that anxiety and depression have been highly prevalent in South Asia, which may have aggravated amid the COVID-19 pandemic due to the continued fear of infection, loss of socioeconomic opportunities, impaired the psychosocial processes, and lack of access to mental health resources and services ${ }^{47,58,72}$. 
COVID-19 pandemic has become a major concern for global mental health, which is evident in a growing body of literature examining how this pandemic has impacted mental health and wellbeing in different contexts and populations ${ }^{6-8,40}$. The findings of the current review can be compared with the existing reviews that synthesized empirical evidence in other geographic regions and population groups. For example, a meta-analysis of 13 studies reported that the prevalence of anxiety and depression in healthcare providers was $23.2 \%$ and $22.8 \%$, respectively. However, this review did not report any studies from South Asian countries. Another metaanalysis of 17 studies assessed mental health problems in general population during COVID-19 and found the prevalence of anxiety and depression as $31.9 \%$ and $33.7 \%$, respectively ${ }^{6}$. Our review found a comparatively higher prevalence through a quantitative synthesis of 35 studies with samples representing healthcare providers as well as the general population in South Asia. The key findings of this review suggest a high burden of depression and anxiety in this region, which necessitates further research to elucidate the psychosocial impacts of COVID-19 in South Asian countries.

\subsection{Implications for future research}

As this review synthesized evidence from cross-sectional studies with any sample size, future research should emphasize on longitudinal studies involving large samples that may provide more generalizable evidence on mental health among South Asian populations. Moreover, 29 out of the 35 included studies deployed an online approach for recruiting study participants and collecting data. Moreover, South Asian countries have a profound digital divide ${ }^{82,83}$; therefore, people who do not use digital devices or services may not be able to participate in surveys that are administered through internet. Furthermore, rural and marginalized communities may have limited access to digital resources that may systematically exclude them from online participation. Therefore, the current studies may not have included a mass proportion of the general public in respective contexts. Future research should engage different population groups using multipronged sampling and recruitment strategies, ensuring social distancing and infection prevention ${ }^{9}$, which may offer the safety of the participants as well as improve the representativeness of those studies. 
The use of different scales and cut-off values may impact prevalence estimates ${ }^{7}$, which informs the need for using validated scales with uniform cut-off values across populations. It is essential to re-evaluate the items and latent constructs in each scale so that common scales with high reliability and validity can be widely used. Nonetheless, back calculations of different scales or sub-scales can be done if the primarily collected data are publicly available for re-analyses. Psychiatric epidemiological research should adhere to data sharing policies that promote transparency and the use of uniform measures across studies. Such measures are likely to provide better estimations of the epidemiological burden of anxiety and depression at the population level.

In this review, most studies recruited participants with varying educational and occupational levels. However, limited sociodemographic data may not show how people of different levels of education or income may experience psychosocial stressors during this pandemic. For example, a higher prevalence of anxiety among the healthcare providers may reflect the occupational challenges and lived experiences of the fear of COVID-19, whereas unemployed people with education may have altered levels of depression ${ }^{8,9,40}$. As COVID-19 has impacted socioeconomic activities in most contexts, such disruptions are likely to affect mental health and wellbeing among the affected occupational groups ${ }^{84}$. In future research, participant-level data with temporal variations in psychosocial measures should be recorded to assess how anxiety and depression may have manifested in different subgroups within the South Asian population.

The existing studies and their syntheses show a high prevalence of anxiety and depression among women in South Asia. Future studies with better participation of women are critical to examine the psychological issues and their determinants that may be associated with gender norms and roles within the context of South Asian countries. Women in these countries have limited autonomy, empowerment, participation in socioeconomic activities, and access to mental health services and resources ${ }^{85}$. It is not clear how preventive measures such as quarantine or lockdown may have affected women's mental health in South Asia. Moreover, gender-based violence is highly prevalent in South Asian countries ${ }^{86,87}$. Previous research has shown tremendous impacts of oppression and violence on women's psychosocial health ${ }^{85,87,88}$. Recent reports suggest a growing burden of domestic violence, which is likely to affect the mental health and wellbeing among South Asian women ${ }^{89}$. Future research should investigate 
such issues that may not only explore the risk factors of depression and anxiety, but also examine social epidemiology of gender-based violence that may affect immediate and long-term mental health outcomes among women.

This review did not limit the age of the study participants; however, limited evidence on the children or older adults suggests a critical research gap during this pandemic. This gap can be attributable to the sampling strategies (e.g., convenient sampling) and recruitment methods (e.g., circulating survey link through social media) that are likely to engage the young participants rather than children and older adults. Further research is needed to examine mental health status in different age groups that may allow a comparative analysis of psychosocial impacts of this pandemic in respective populations ${ }^{90,91}$. Moreover, online surveys may engage informal and family caregivers who may facilitate the participation of the children or older adults they live with, which may address the current knowledge gaps on those individuals.

While most studies in this review focused on the general population and healthcare providers, there is a lack of evidence on the prevalence of anxiety and depression among COVID-19 patients and their family caregivers. A study conducted among COVID-19 patients in North India found 62.9\% prevalence of depression, suggesting a higher psychological burden in this population ${ }^{67}$. Prospective studies in South Asian countries should focus on these individuals, who may have experienced severe psychosocial distress and adverse mental health conditions during this pandemic.

People with chronic conditions are likely to have adverse health outcomes during COVID-19, which may increase psychological distress and deteriorated mental health outcomes in this pandemic ${ }^{6,9}$. Also, people with chronic mental disorders may have been disproportionately affected during this pandemic who may have lost access to care and other services. Moreover, coexisting mental disorders among the same individuals may affect the overall health and quality of living ${ }^{71,79,92,93}$. Future research should pay special attentions to investigate anxiety, depression, and other mental health problems among those vulnerable individuals and populations. 
Media use and quality of media contents may affect the mental health of individuals and populations amid this pandemic ${ }^{15}$. Misinformation and disinformation from available media sources may create psychological distress, whereas the lack of access to reliable sources may leave individuals with uncertainties regarding this pandemic. Moreover, people confined at home or those with altered workstyle from home may have been exposed to excessive digital media time or screen use behavior that may influence their mental health ${ }^{94,95}$. Further research is needed to clarify how the type, duration, contents, and quality of media use can influence anxiety, depression, and other mental health outcomes in South Asian populations.

It is necessary to investigate the social determinants of mental health in South Asia and their trajectories during and after this pandemic. An altered psychosocial state may impact intrapersonal, interpersonal, and community-based psychological processes and outcomes in the population level ${ }^{54,72}$. Moreover, systematic oppression and repression in socioeconomically marginalized individuals may adversely influence the biopsychosocial processes, resulting in a higher burden of depression and other mental health problems. The high prevalence of anxiety and depression in this review informs the need for extensive research on such determinants among South Asian populations, which may facilitate preventive measures addressing those underlying disparities.

Implementation research is a neglected domain in the realm of global mental health. In the context of South Asia, little is known about how mental health policies, programs, and other population-level measures are developed, implemented, and evaluated ${ }^{33,96}$. Nonetheless, the scope of mental health services within the current pandemic preparedness plans is yet to be examined. Policy researchers should complement epidemiological investigations through assessing the relevance of evidence-based public mental health interventions in the context of South Asia. Moreover, implementation research outcomes should be widely communicated to foster timely decision-making and setting priorities for future research and actions.

South Asian countries have a comparative lack of empirical research on mental health problems despite having a large proportion of the global population ${ }^{37,38,97}$. This was revalidated by the low number of empirical studies in the current review. Moreover, most studies were from India, Bangladesh, and Pakistan, with fewer studies from Nepal and Sri Lanka. No study was identified from Afghanistan, Bhutan, and Maldives, highlighting a research disparity in this region. 
Moreover, studies from one country may not recruit nationally representative samples, which may affect the generalizability of the subgroup estimates. These research gaps within and between the South Asian countries can be attributable to several issues such as a lack of research funding, inadequate institutional capacities, a low demand of scientific evidence among the key decision-makers, and other context-specific factors in respective health systems ${ }^{37,38}$. It is necessary to address these challenges through engaging institutional stakeholders and empowering them to use research data for mental health policymaking. Stewardship approaches should be adopted by the healthcare leadership to strengthen country-level and regional research capacities and collaborations, which may improve the knowledge base amid this pandemic as well as advance mental health policymaking and practice.

\subsection{Implications for mental health policymaking and practice}

The current evidence suggests a high prevalence of anxiety and depression in South Asian countries, which requires increasing availability of and accessibility to mental health services. Such measures are critical to diagnose anxiety and depression in high-risk populations and deliver psychiatric and psychological therapies to the affected individuals. However, South Asian countries have a serious scarcity of mental health professionals according to the prepandemic estimates ${ }^{33,38,96}$, which may aggravate during this pandemic as many healthcare organizations are prioritizing infection prevention and in-patient services for physical health problems. This growing gap should be acknowledged and addressed using contextually appropriate actions that may improve access to mental health services alongside pandemic control in respective populations. Potential strategies to improve mental health services during this pandemic may include task-shifting of mental health services through capacity building, initiating telepsychiatric care services, incentivizing healthcare providers and organizations for facilitating mental health services, and strengthening mental health systems for delivering mental health in the target populations ${ }^{9,14,84,98,99}$.

This review suggests a relatively higher burden of anxiety and depression among marginalized populations groups such as women and occupational groups with higher psychological distress such as healthcare providers. It is essential to adopt targeted interventions for high-risk groups. 
Some of these interventions may be delivered in workplaces or organizations, whereas mass media interventions or self-management resources can be delivered in a more generalized manner targeting a broader audience ${ }^{9,84}$. Such efforts should promote early diagnosis and referral to specialized care for individuals with advanced symptoms. Moreover, stigma to mental health problems is a critical psychosocial challenge in South Asia ${ }^{38,96}$. Socio-culturally appropriate media campaigns should be organized to sensitize the general population, address stigma, and encourage them to participate in mental health programs.

In recent years, digital mental health has emerged as a promising approach to improve access to mental health services using digital devices and internet-based technologies ${ }^{100}$. Such interventions may facilitate self-management and patient-provider communication in mental health practice. However, the evidence on the effectiveness of such interventions during this pandemic is not well known ${ }^{101}$. Previous research suggests a lack of digital mental health interventions in South Asian countries ${ }^{83}$, therefore, conceptualizing and delivering such interventions during this pandemic would involve expert opinions and the evidence available from other contexts. These initiatives should engage patients, caregivers, and mental healthcare providers to incorporate their perspectives, needs, and recommendations to optimize future digital interventions in the realm of mental health.

Primary care and community-based health facilities play a vital role in delivering preventive and therapeutic health services in South Asian countries ${ }^{102-104}$. However, the current capacity of such community-level facilities to deliver mental health services during this pandemic may not be adequate to address the continued burden of mental disorders among the communities they serve ${ }^{33,96}$. Mental health policymakers and practitioners may need to assess the preparedness of these facilities and explore potential opportunities to strengthen community-based, low-cost, evidence-based, and sustainable mental health services during COVID-19 pandemic.

The current evidence suggests the need for accurate information and measures for preventing COVID-19 pandemic ${ }^{15,51,105}$, which may address psychosocial distress among individuals and populations. Online and mass media health communication interventions should be developed in cooperation with media stakeholders and public health agencies ensuring effective risk communication during this pandemic. Moreover, healthcare providers should be equipped with 
protective equipment and psychoeducational resources that may address mental health problems due to occupational stressors ${ }^{106-108}$.

Health and socioeconomic consequences of the COVID-19 pandemic may have disrupted public mental health programs in South Asian countries, which is a major challenge for providing mental health during this crisis as well as restoring the program activities for achieving program goals and targets. For example, India has National Mental Health Program and District Mental Health program that target population mental health, whereas multicomponent programs such as Rashtriya Kishor Swasthya Karyakram (RKSK) aims to improve mental health and wellbeing in adolescents alongside overall health promotion ${ }^{33}$. Since the institutional points of care such as the schools or community outreach events may not remain operational during this pandemic, those mental health programs may not be delivered to the target populations as they have been planned previously. These programs require careful attention and proactive efforts to revitalize the same. Moreover, restoring public mental health programs should be consistent with infection control measures and prevention policies in respective contexts.

Strengthening mental health services and programs may require resources that are often facilitated through the provisions of mental health policies in respective countries. However, South Asian countries have policy-level gaps in terms of coordinated mental health service delivery that are well documented ${ }^{38,96}$. Moreover, local and national mental health policies may not have scopes for mobilizing resources in complex situations and public health emergencies. Two major approaches can be recommended in this regard. First, revisiting the current policies and leveraging available resources to facilitate mental health services. Second, adopting standalone mental health programs for addressing mental health problems during and after this pandemic that may complement the existing policies and programs. Devising policy alternatives can be a lengthy process, which is a common obstacle for decision-making during public health emergencies. A rapid evidence synthesis should be conducted, and all stakeholders should be informed regarding the available strategies that can be enacted, minimizing the potential misuse of resources and maximizing mental health actions.

Lastly, COVID-19 has deeply impacted every aspects of human lives and widened health disparities across contexts, which necessitates a holistic approach to address the psychosocial and environmental determinants of mental disorders ${ }^{9,46,54}$. Most of the South Asian countries 
have a high burden of social diseases such as poverty, unemployment, illiteracy, gender inequity, interpersonal violence, political unrest, systematic exclusion of minorities from socioeconomic opportunities, and many other issues that may adversely affect mental health and wellbeing across population groups ${ }^{27,37,38,85}$. Such problems are likely to accelerate amid the changing socioeconomic landscape during the COVID-19 pandemic and increase the psychosocial stressors resulting in a growing burden of mental health problems. The coexistence of and interactions between multiple biophysical and psychosocial problems are suggestive of a syndemic ${ }^{109}$, which may have long term population health consequences in this region. The policymakers and other key stakeholders must acknowledge and address these complex problems to improve public mental health in South Asian countries. In addition, public institutions and regulatory authorities should reach a consensus to develop mental health resilience through multipronged social efforts at the population level. Furthermore, collaborative decision-making may empower communities and institutions to adopt evidencebased, integrated, and timely measures that are necessary to alleviate the psychosocial burden of COVID-19 in South Asia.

\subsection{Limitations of this review}

This meta-analytic review has several limitations. First, a high publication bias indicates that some studies might not have been included in the current review. We focused on peer-reviewed journal sources; therefore, preprints and non-indexed journal articles were beyond the scope of this review. Although this measure ensured a better quality of our review by including peerreviewed evidence only, this might have excluded some studies that could provide further epidemiological insights on anxiety and depression. Second, the use of study-level data may lead to less accurate estimates compared to analyzing participant-level data. Most studies did not include an open data sharing approach that limited the opportunity to examine intra-study and inter-study variations in meta-analyses. Last, we did not find enough data to construct and use multiple variables in additional sub-group analyses and meta-regression that could explain the potential sources of heterogeneity across studies. Moreover, such data could provide further insights on variances in population groups that would inform policymaking and clinical care for high-risk groups. We encourage future researchers to use uniform measurement instruments, 
recruit samples that represent the populations of interest, adopt liberal data sharing approaches, and improve the knowledge base through a more rigorous synthesis of evidence.

\section{Conclusion}

COVID-19 pandemic has adversely affected psychosocial health and wellbeing globally. This review synthesized the quantitative evidence on the prevalence of anxiety and depression in South Asia during this pandemic. The results inform a high burden of anxiety and depression in this region, necessitating the adoption of multilevel mental health interventions alleviating psychosocial burden of COVID-19. Moreover, high heterogeneity among studies and marked knowledge gaps on psychosocial epidemiology in South Asian countries inform the need for further research, which may facilitate evidence-based mental health promotion in this region during and after this pandemic.

Conflicts of interests: None.

Acknowledgment: None.

Funding: No financial support was received at any stage of conducting this review. 


\section{References}

1. World Health Organization. Coronavirus disease (COVID-19).

https://www.who.int/emergencies/diseases/novel-coronavirus-2019. Published 2020. Accessed October 29, 2020.

2. Centers for Disease Control and Prevention. Coronavirus Disease 2019 (COVID-19). https://www.cdc.gov/coronavirus/2019-ncov/cdcresponse/about-COVID-19.html. Published 2020. Accessed October 29, 2020.

3. World Health Organization. WHO Director-General's Opening Remarks at the Media Briefing on COVID-19 - 11 March 2020.; 2020. https://www.who.int/dg/speeches/detail/who-directorgeneral-s-opening-remarks-at-the-media-briefing-on-covid-19---11-march-2020. Accessed October 29, 2020.

4. Chaudhry R, Dranitsaris G, Mubashir T, Bartoszko J, Riazi S. A country level analysis measuring the impact of government actions, country preparedness and socioeconomic factors on COVID-19 mortality and related health outcomes. EClinicalMedicine. 2020;25. doi:10.1016/j.eclinm.2020.100464

5. Tabari P, Amini M, Moghadami M, Moosavi M. International public health responses to COVID-19 outbreak: A rapid review. Iran J Med Sci. 2020;45(3):157-169. doi:10.30476/ijms.2020.85810.1537

6. Salari N, Hosseinian-Far A, Jalali R, et al. Prevalence of stress, anxiety, depression among the general population during the COVID-19 pandemic: A systematic review and metaanalysis. Global Health. 2020;16(1):57. doi:10.1186/s12992-020-00589-w

7. Deng J, Zhou F, Hou W, et al. The prevalence of depression, anxiety, and sleep disturbances in COVID-19 patients: a meta-analysis. Ann N Y Acad Sci. October 2020:nyas.14506. doi:10.1111/nyas.14506

8. Bueno-Notivol J, Gracia-García P, Olaya B, Lasheras I, López-Antón R, Santabárbara J. Prevalence of depression during the COVID-19 outbreak: A meta-analysis of communitybased studies. Int J Clin Heal Psychol. 2020:xxx---xxx. doi:10.1016/j.ijchp.2020.07.007 
9. Hossain MM, Tasnim S, Sultana A, et al. Epidemiology of mental health problems in COVID-19: a review. F1000Research. 2020;9:636. doi:10.12688/f1000research.24457.1

10. Singh S, Roy D, Sinha K, Parveen S, Sharma G, Joshi G. Impact of COVID-19 and lockdown on mental health of children and adolescents: A narrative review with recommendations. Psychiatry Res. 2020;293:113429. doi:10.1016/j.psychres.2020.113429

11. Amerio A, Brambilla A, Morganti A, et al. COVID-19 Lockdown: Housing Built Environment's Effects on Mental Health. Int J Environ Res Public Health. 2020;17(16):5973. doi:10.3390/ijerph17165973

12. Webb L. COVID-19 lockdown: A perfect storm for older people's mental health. J Psychiatr Ment Health Nurs. June 2020:jpm.12644. doi:10.1111/jpm.12644

13. Rossi R, Socci V, Talevi D, et al. COVID-19 Pandemic and Lockdown Measures Impact on Mental Health Among the General Population in Italy. Front Psychiatry. 2020;11:790. doi:10.3389/fpsyt.2020.00790

14. Hossain MM, Sultana A, Purohit N. Mental health outcomes of quarantine and isolation for infection prevention: a systematic umbrella review of the global evidence. Epidemiol Health. 2020;42. doi:10.4178/epih.e2020038

15. Tasnim S, Hossain M, Mazumder H. Impact of rumors and misinformation on COVID-19 in Social Media. J Prev Med Public Heal. 2020;53(3):171-174. doi:10.3961/JPMPH.20.094

16. Chong MY, Wang WC, Hsieh WC, et al. Psychological impact of severe acute respiratory syndrome on health workers in a tertiary hospital. Br J Psychiatry. 2004;185(AUG.):127133. doi:10.1192/bjp.185.2.127

17. Wu P, Fang Y, Guan Z, et al. The psychological impact of the SARS epidemic on hospital employees in China: Exposure, risk perception, and altruistic acceptance of risk. Can J Psychiatry. 2009;54(5):302-311. doi:10.1177/070674370905400504

18. Yip PSF, Cheung YT, Chau PH, Law YW. The impact of epidemic outbreak: The case of severe acute respiratory syndrome (SARS) and suicide among older adults in Hong Kong. Crisis. 2010;31(2):86-92. doi:10.1027/0227-5910/a000015 
19. Chew QH, Wei KC, Vasoo S, Chua HC, Sim K. Narrative synthesis of psychological and coping responses towards emerging infectious disease outbreaks in the general population: Practical considerations for the COVID-19 pandemic. Singapore Med J. 2020;61(7):350-356. doi:10.11622/SMEDJ.2020046

20. da Silva DAR, Weyll Pimentel RF, das Merces MC. Covid-19 and the pandemic of fear: Reflections on mental health. Rev Saude Publica. 2020;54. doi:10.11606/S15188787.2020054002486

21. Chi X, Becker B, Yu Q, et al. Prevalence and Psychosocial Correlates of Mental Health Outcomes Among Chinese College Students During the Coronavirus Disease (COVID-19) Pandemic. Front Psychiatry. 2020;11:1. doi:10.3389/fpsyt.2020.00803

22. Wang C, Pan R, Wan X, et al. Immediate psychological responses and associated factors during the initial stage of the 2019 coronavirus disease (COVID-19) epidemic among the general population in China. Int J Environ Res Public Health. 2020;17(5):1729. doi:10.3390/ijerph17051729

23. Chi X, Becker B, Yu Q, et al. Persistence and remission of depressive symptoms and psycho-social correlates in Chinese early adolescents. BMC Psychiatry. 2020;20(1):406. doi:10.1186/s12888-020-02808-5

24. Qiu J, Shen B, Zhao M, Wang Z, Xie B, Xu Y. A nationwide survey of psychological distress among Chinese people in the COVID-19 epidemic: Implications and policy recommendations. Gen Psychiatry. 2020;33(2). doi:10.1136/gpsych-2020-100213

25. Tasnim S, Rahman M, Pawar P, et al. Epidemiology of sleep disorders during COVID-19 pandemic: A systematic scoping review. medRxiv. October 2020:2020.10.08.20209148. doi:10.1101/2020.10.08.20209148

26. Bhutta ZA, Basnyat B, Saha S, Laxminarayan R. Covid-19 risks and response in South Asia. BMJ. 2020;368. doi:10.1136/bmj.m1190

27. Roy A, Singh AK, Mishra S, Chinnadurai A, Mitra A, Bakshi O. Mental health implications of COVID-19 pandemic and its response in India. Int J Soc Psychiatry. 2020. doi:10.1177/0020764020950769 
28. Galea S, Merchant RM, Lurie N. The Mental Health Consequences of COVID-19 and Physical Distancing: The Need for Prevention and Early Intervention. JAMA Intern Med. 2020;180(6):817-818. doi:10.1001/jamainternmed.2020.1562

29. Rahman A, Naslund JA, Betancourt TS, et al. The NIMH global mental health research community and COVID-19. The Lancet Psychiatry. 2020;7(10):834-836. doi:10.1016/S22150366(20)30347-3

30. Kar SK, Yasir Arafat SM, Kabir R, Sharma P, Saxena SK. Coping with Mental Health Challenges During COVID-19. In: Coronavirus Disease 2019 (COVID-19). Nature Publishing Group; 2020:199-213. doi:10.1007/978-981-15-4814-7_16

31. World Health Organization. COVID-19 disrupting mental health services in most countries, WHO survey. https://www.who.int/news/item/05-10-2020-covid-19disrupting-mental-health-services-in-most-countries-who-survey. Published 2020. Accessed October 29, 2020.

32. Mubbashar MH, Saeed K. Development of mental health services in Pakistan. East Mediterr Heal J. 2001;7(3):392-396. doi:10.1192/s1749367600007633

33. Hossain MM, Purohit N. Improving child and adolescent mental health in India: Status, services, policies, and way forward. Indian J Psychiatry. 2019;61(4):415-419. doi:10.4103/psychiatry.IndianJPsychiatry_217_18

34. Desai NG, Tiwari SC, Nambi S, et al. Urban mental health services in India : how complete or incomplete? Indian J Psychiatry. 2004;46(3):195-212. http://www.ncbi.nlm.nih.gov/pubmed/21224901. Accessed October 29, 2020.

35. Hossain MM, Hasan MT, Sultana A, Faizah F. New Mental Health Act in Bangladesh: unfinished agendas. The Lancet Psychiatry. 2019;6(1):e1. doi:10.1016/S2215-0366(18)304723

36. Naveed S, Waqas A, Chaudhary AMD, et al. Prevalence of Common Mental Disorders in South Asia: A Systematic Review and Meta-Regression Analysis. Front Psychiatry. 2020;11:899. doi:10.3389/fpsyt.2020.573150 
37. Hossain MM, Purohit N, Sultana A, Ma P, Mckyer LJ, Ahmed U. Prevalence of mental disorders in South Asia: An umbrella review of systematic reviews and meta-analyses. Asian J Psychiatr. 2020. doi:10.1016/j.ajp.2020.102041

38. Trivedi JK, Goel D, Kallivayalil RA, Isaac M, Shrestha DM, Gambheera HC. Regional cooperation in South Asia in the field of mental health. World Psychiatry. 2007;6(1):57-59. http://www.ncbi.nlm.nih.gov/pubmed/17342229. Accessed October 29, 2020.

39. Liberati A, Altman DG, Tetzlaff J, et al. The PRISMA statement for reporting systematic reviews and meta-analyses of studies that evaluate healthcare interventions: explanation and elaboration. BMJ. 2009;339. doi:10.1136/bmj.b2700

40. Pappa S, Ntella V, Giannakas T, Giannakoulis VG, Papoutsi E, Katsaounou P. Prevalence of depression, anxiety, and insomnia among healthcare workers during the COVID-19 pandemic: A systematic review and meta-analysis. Brain Behav Immun. 2020;88:901-907. doi:10.1016/j.bbi.2020.05.026

41. Wallace BC, Dahabreh IJ, Trikalinos TA, Lau J, Trow P, Schmid CH. Closing the gap between methodologists and end-users: R as a computational back-end. J Stat Softw. 2012;49(1):1-15. doi:10.18637/jss.v049.i05

42. Freemantle N. StatsDirect-Statistical Software for Medical Research in the 21st Century. BMJ. 2000;321(7275):1536. doi:10.1136/bmj.321.7275.1536

43. Barendregt JJ, Doi SA, Lee YY, Norman RE, Vos T. Meta-analysis of prevalence. J Epidemiol Community Health. 2013;67(11):974-978. doi:10.1136/jech-2013-203104

44. DerSimonian R, Laird N. Meta-analysis in clinical trials. Control Clin Trials. 1986;7(3):177188. doi:10.1016/0197-2456(86)90046-2

45. Higgins JPT, Thompson SG, Deeks JJ, Altman DG. Measuring inconsistency in metaanalyses. Br Med J. 2003;327(7414):557-560. doi:10.1136/bmj.327.7414.557

46. Ahmad A, Rahman I, Agarwal M. Early psychosocial predictors of mental health among Indians during coronavirus disease 2019 outbreak. J Heal Sci. 2020;10(2):147-156. doi:10.17532/jhsci.2020.950 
47. Ahmed O, Ahmed MZ, Alim SMAHM, Khan MDAU, Jobe MC. COVID-19 outbreak in Bangladesh and associated psychological problems: An online survey. Death Stud. 2020;0(0):1-10. doi:10.1080/07481187.2020.1818884

48. Gupta S, Kohli K, Padmakumari P, et al. Psychological Health Among Armed Forces Doctors During COVID-19 Pandemic in India. Indian J Psychol Med. 2020;42(4):374-378. doi:10.1177/0253717620934037

49. Gupta S, Prasad AS, Dixit PK, Padmakumari P, Gupta S, Abhisheka K. Survey of prevalence of anxiety and depressive symptoms among 1124 healthcare workers during the coronavirus disease 2019 pandemic across India. Med J Armed Forces India. 2020. doi:10.1016/j.mjafi.2020.07.006

50. Hasan SR, Hamid Z, Jawaid MT, Ali RK. Anxiety among doctors during COVID-19 pandemic in secondary and tertiary care hospitals. Pakistan J Med Sci. 2020;36(6):13601365. doi:10.12669/pjms.36.6.3113

51. Hossain T, Ahammed B, Chanda SK, Jahan N, Ela MZ, Islam N. Social and electronic media exposure and generalized anxiety disorder among people during COVID-19 outbreak in Bangladesh: A preliminary observation. PLoS One. 2020;15(9 September):1-13. doi:10.1371/journal.pone.0238974

52. Imran N, Masood HMU, Ayub M, Gondal KM. Psychological impact of COVID-19 pandemic on postgraduate trainees: a cross-sectional survey. Postgrad Med J. 2020:postgradmedj-2020-138364. doi:10.1136/postgradmedj-2020-138364

53. Imran N, Hashmi AM, Masud K. The Toll it Takes: Mental Health Burden and Associated Factors During COVID-19 Outbreak among Healthcare Workers in Lahore, Pakistan. Ann King Edward Med Univ. 2020;26(2):317-323.

54. Akhtarul Islam M, Barna SD, Raihan H, Nafiul Alam Khan M, Tanvir Hossain M. Depression and anxiety among university students during the COVID-19 pandemic in Bangladesh: A web-based cross-sectional survey. PLoS One. 2020;15(8 August):1-12. doi:10.1371/journal.pone.0238162

55. Islam MS, Ferdous MZ, Potenza MN. Panic and generalized anxiety during the COVID-19 
pandemic among Bangladeshi people: An online pilot survey early in the outbreak. $J$ Affect Disord. 2020;276(July):30-37. doi:10.1016/j.jad.2020.06.049

56. Jain A, Singariya G, Kamal M, Kumar M, Jain A, Solanki R. COVID-19 pandemic: Psychological impact on anaesthesiologists. Indian J Anaesth. 2020;64(9):774-774. https://go.gale.com/ps/i.do?p=AONE\&sw=w\&issn=00195049\&v=2.1\&it=r\&id=GALE\%7CA 634738196\&sid=googleScholar\&linkaccess=fulltext. Accessed October 24, 2020.

57. Khanna R, Honavar S, Metla A, Bhattacharya A, Maulik P. Psychological impact of COVID-19 on ophthalmologists-in-training and practising ophthalmologists in India. Indian J Ophthalmol. 2020;68(6):994. doi:10.4103/ijo.IJO_1458_20

58. Amin F, Sharif S, Saeed R, Durrani N, Jilani D. COVID-19 pandemic- knowledge, perception, anxiety and depression among frontline doctors of Pakistan. BMC Psychiatry. 2020;20(1):459. doi:10.1186/s12888-020-02864-x

59. Khanal P, Devkota N, Dahal M, Paudel K, Joshi D. Mental health impacts among health workers during COVID-19 in a low resource setting: a cross-sectional survey from Nepal. Global Health. 2020;16(1):89. doi:10.1186/s12992-020-00621-z

60. Mamun MA, Sakib N, Gozal D, et al. The COVID-19 pandemic and serious psychological consequences in Bangladesh: a population-based nationwide study. J Affect Disord. October 2020. doi:10.1016/j.jad.2020.10.036

61. Mani VE, Sarkar Z, Gutti NB. A Study of the Impact of the COVID-19 Pandemic on Anxiety Levels of Young Adults in India. J Evol Med Dent Sci. 2020;9(31):2233-2238. doi:10.14260/jemds/2020/485

62. Nihmath Nisha S, Francis YM, Balaji K, Raghunath G, Kumaresan M. A survey on anxiety and depression level among South Indian medical students during the COVID 19 pandemic. Int J Res Pharm Sci. 2020;11(Special Issue 1):779-786. doi:10.26452/ijrps.v11iSPL1.3082

63. Patabendige M, Gamage MM, Weerasinghe M, Jayawardane A. Psychological impact of the COVID-19 pandemic among pregnant women in Sri Lanka. Int J Gynecol Obstet. 2020;151(1):150-153. doi:10.1002/ijgo.13335 
64. Reddy V V, Revanth Karri S, Jezreel T, Afeen S, Khairkar P. Psychosocial Impact of COVID-19 Lockdown on Mental Wellbeing among 11 States of India: A Markov Modeling Approach. J Psychiatry Psychiatr Disord. 2020;04(04):158-174. doi:10.26502/jppd.2572519x0103

65. Sandesh R, Shahid W, Dev K, et al. Impact of COVID-19 on the Mental Health of Healthcare Professionals in Pakistan. Cureus. 2020;12(7):3-7. doi:10.7759/cureus.8974

66. Sebastian J, Anand A, Vakkalaganti Rajesh R, Lucca JM, Joseph R. Impact of covid-19 pandemic on psychological responses of the general population in india: A nationwide survey | International Journal of Pharmaceutical Research;12:2349-2357, 2020. | COVIDWHO. Int J Pharm Res. 2020;12:2349-2357. http://www.ijpronline.com/ViewSpecialArticleDetail.aspx?ID=486. Accessed October 24, 2020.

67. Sharma S, Bansal R, Parashar P, Ahmad S, Kumar A, Kumar A. Stress and Depression Among People Admitted in a COVID 19 Quarantine Centre of North India. Subharti J Interdiscip Res. 2020;3.

68. Shrestha SL. Prevalence of psychological effect of COVID-19 on medical professionals in a tertiary care center. J Nepal Med Assoc. 2020;58(228):550-553. doi:10.31729/jnma.5087

69. Banna MH Al, Sayeed A, Kundu S, et al. The impact of the COVID-19 pandemic on the mental health of the adult population in Bangladesh: a nationwide cross-sectional study. Int J Environ Health Res. 2020;00(00):1-12. doi:10.1080/09603123.2020.1802409

70. Sil A, Das A, Jaiswal S, et al. Mental health assessment of frontline COVID-19 dermatologists: A Pan-Indian multicentric cross-sectional study. Dermatol Ther. 2020;(June):8-9. doi:10.1111/dth.13884

71. Suryavanshi N, Kadam A, Dhumal G, et al. Mental health and quality of life among healthcare professionals during the COVID-19 pandemic in India. Brain Behav. 2020;(July):1-12. doi:10.1002/brb3.1837

72. Verma S, Mishra A. Depression, anxiety, and stress and socio-demographic correlates among general Indian public during COVID-19. Int J Soc Psychiatry. 2020;66(8):756-762. 
doi:10.1177/0020764020934508

73. Wilson W, Raj JP, Rao S, et al. Prevalence and Predictors of Stress, anxiety, and Depression among Healthcare Workers Managing COVID-19 Pandemic in India: A Nationwide Observational Study. Indian J Psychol Med. 2020;42(4):353-358. doi:10.1177/0253717620933992

74. Zubayer A Al, Rahman ME, Islam MB, et al. Psychological states of Bangladeshi people four months after the COVID-19 pandemic: An online survey. Heliyon. 2020;6(9):e05057. doi:10.1016/j.heliyon.2020.e05057

75. Chatterjee S, Bhattacharyya R, Bhattacharyya S, Gupta S, Das S, Banerjee B. Attitude, practice, behavior, and mental health impact of COVID-19 on doctors. Indian J Psychiatry. 2020;62(3):257. doi:10.4103/psychiatry.IndianJPsychiatry_333_20

76. Chew NWS, Lee GKH, Tan BYQ, et al. A multinational, multicentre study on the psychological outcomes and associated physical symptoms amongst healthcare workers during COVID-19 outbreak. Brain Behav Immun. 2020;88(April):559-565. doi:10.1016/j.bbi.2020.04.049

77. Chew NWS, Ngiam JN, Tan BY-Q, et al. Asian-Pacific perspective on the psychological well-being of healthcare workers during the evolution of the COVID-19 pandemic. BJPsych open. 2020;6(6):e116. doi:10.1192/bjo.2020.98

78. Desai MZJH, Khan AR, Kulkarni R, Hegde B. The psychological impact of COVID-19 and the subsequent social isolation on the general population of Karnataka, India.J Ideas Heal. 2020;3(Special1):190-195. doi:10.47108/jidhealth.vol3.issspecial1.51

79. Grover S, Sahoo S, Mehra A, et al. Psychological impact of COVID-19 lockdown: An online survey from India. Indian J Psychiatry. 2020;62(4):354. doi:10.4103/psychiatry.indianjpsychiatry_427_20

80. Gupta AK, Mehra A, Niraula A, et al. Prevalence of anxiety and depression among the healthcare workers in Nepal during the COVID-19 pandemic. Asian J Psychiatr. 2020;54(January). doi:10.1016/j.ajp.2020.102260 
81. Hossain MM. Current status of global research on novel coronavirus disease (COVID-19): a bibliometric analysis and knowledge mapping. F1000Research. 2020;9:374. doi:10.12688/f1000research.23690.1

82. Zhou Y, Singh N, Kaushik PD. The digital divide in rural South Asia: Survey evidence from Bangladesh, Nepal and Sri Lanka. IIMB Manag Rev. 2011;23(1):15-29. doi:10.1016/j.iimb.2010.12.002

83. Hossain MM, Tasnim S, Sharma R, et al. Digital interventions for people living with noncommunicable diseases in India: A systematic review of intervention studies and recommendations for future research and development. Digit Heal. 2019;5:205520761989615. doi:10.1177/2055207619896153

84. Holmes EA, O'Connor RC, Perry VH, et al. Multidisciplinary research priorities for the COVID-19 pandemic: a call for action for mental health science. The Lancet Psychiatry. 2020;7(6):547-560. doi:10.1016/S2215-0366(20)30168-1

85. Niaz U, Hassan S. Culture and mental health of women in South-East Asia. World Psychiatry. 2006;5(2):118-120. http://www.ncbi.nlm.nih.gov/pubmed/16946955. Accessed October 29, 2020.

86. Hossain MM, Sultana A, Das A. Gender-based violence among Rohingya refugees in Bangladesh: a public health challenge. Indian J Med Ethics. 2018. doi:10.20529/ijme.2018.045

87. Jejeebhoy SJ, Santhya KG, Acharya R. Violence against women in South Asia: The need for the active engagement of the health sector. Glob Public Health. 2014;9(6):678-690. doi:10.1080/17441692.2014.916736

88. Keynejad RC, Hanlon C, Howard LM. Psychological interventions for common mental disorders in women experiencing intimate partner violence in low-income and middleincome countries: a systematic review and meta-analysis. The Lancet Psychiatry. 2020;7(2):173-190. doi:10.1016/S2215-0366(19)30510-3

89. vora M, Malathesh BC, Das S, Chatterjee SS. COVID-19 and domestic violence against women. Asian J Psychiatr. 2020;53:102227. doi:10.1016/j.ajp.2020.102227 
90. Sharma R, Hossain MM, Pawar P, Sharma S. Air pollution and geriatric mental health: Perspectives on the COVID-19 pandemic. Int Psychogeriatrics. 2020:1-2. doi:10.1017/S1041610220001428

91. Mazumder H, Hossain MM, Das A. Geriatric Care during Public Health Emergencies: Lessons Learned from Novel Corona Virus Disease (COVID-19) Pandemic. J Gerontol Soc Work. 2020;63(4):257-258. doi:10.1080/01634372.2020.1746723

92. Bhalla IP, Stefanovics EA, Rosenheck RA. Mental health multimorbidity and poor quality of life in patients with schizophrenia. Schizophr Res. 2018;201:39-45. doi:10.1016/j.schres.2018.04.035

93. Hossain MM, Khan N, Sultana A, et al. Prevalence of comorbid psychiatric disorders among people with autism spectrum disorder: An umbrella review of systematic reviews and meta-analyses. Psychiatry Res. 2020;287. doi:10.1016/j.psychres.2020.112922

94. Smith L, Jacob L, Trott M, et al. The association between screen time and mental health during COVID-19: A cross sectional study. Psychiatry Res. 2020;292:113333. doi:10.1016/j.psychres.2020.113333

95. Sultana A, Tasnim S, Bhattacharya S, Hossain MM, Purohit N. Digital screen time during COVID-19 pandemic: A public health concern. SocArXiv. 2020. doi:10.31235/OSF.IO/E8SG7

96. Shidhaye R, Sikander S, Jordans M, De Silva P, Chatterjee S. Mental Health Programs and Policies in South Asia: Initiatives and Obstacles. In: Mental Health in South Asia: Ethics, Resources, Programs and Legislation. Springer, Dordrecht; 2015:95-111. doi:10.1007/978-94017-9017-8_7

97. Hossain MM, Sultana A, Tasnim S, et al. Prevalence of mental disorders among people who are homeless: An umbrella review. Int J Soc Psychiatry. 2020;66(6):528-541. doi:10.1177/0020764020924689

98. Xiang YT, Yang Y, Li W, et al. Timely mental health care for the 2019 novel coronavirus outbreak is urgently needed. The Lancet Psychiatry. 2020;7(3):228-229. doi:10.1016/S22150366(20)30046-8 
99. Duan L, Zhu G. Psychological interventions for people affected by the COVID-19 epidemic. The Lancet Psychiatry. 2020;7(4):300-302. doi:10.1016/S2215-0366(20)30073-0

100. Torous J, Myrick KJ, Rauseo-Ricupero N, Firth J. Digital mental health and COVID-19: Using technology today to accelerate the curve on access and quality tomorrow. J Med Internet Res. 2020;22(3). doi:10.2196/18848

101. Soron TR, Shariful Islam SM, Ahmed HU, Ahmed SI. The hope and hype of telepsychiatry during the COVID-19 pandemic. The Lancet Psychiatry. 2020;7(8):e50. doi:10.1016/S22150366(20)30260-1

102. Hossain MM, Sultana A, Munzur-E-Murshid. Revitalising general practice in Bangladesh: Complementing 'the Bangladesh Paradox. Br J Gen Pract. 2018;68(675):482. doi:10.3399/bjgp18X699173

103. Sultana A, Bhattacharya S, Hossain MM. COVID-19 and primary care: A critical need for strengthening emergency preparedness across health systems. SocArXiv. 2020. doi:10.31235/OSF.IO/BWS84

104. Bhattacharya S, Juyal R, Hossain M, Singh A. Non-communicable diseases viewed as "collateral damage" of our decisions: Fixing accountabilities and finding sloutions in primary care settings. J Fam Med Prim Care. 2020;9(5):2176. doi:10.4103/jfmpc.jfmpc_202_20

105. Bhattacharya S, Sharma N, Hoedebecke K, Hossain M, Gökdemir Ö, Singh A. Harnessing the potential of uploading health educational materials on medical institutions' social media for controlling emerging and re-emerging disease outbreaks. J Educ Health Promot. 2020;9(1):213. doi:10.4103/jehp.jehp_215_20

106. Bhattacharya S, Singh A, Hossain M. Health system strengthening through Massive Open Online Courses (MOOCs) during the COVID-19 pandemic: An analysis from the available evidence.J Educ Health Promot. 2020;9(1):195. doi:10.4103/jehp.jehp_377_20

107. Sultana A, Sharma R, Hossain MM, Bhattacharya S, Purohit N. Burnout among healthcare providers during COVID-19: Challenges and evidence-based interventions. Indian J Med Ethics. 2020:01-04. doi:10.20529/IJME.2020.73 
108. Bhattacharya S, Hossain MM, Singh A. Addressing the shortage of personal protective equipment during the COVID-19 pandemic in India-A public health perspective. AIMS Public Heal. 2020;7(2):223-227. doi:10.3934/publichealth.2020019

109. Gravlee CC. Systemic racism, chronic health inequities, and COVID-19: A syndemic in the making? Am J Hum Biol. 2020;32(5). doi:10.1002/ajhb.23482 
Supplementary file- A: Risk of bias assessment

\begin{tabular}{|c|c|c|c|c|c|c|c|c|}
\hline $\begin{array}{l}\text { Author (Last } \\
\text { name., et al.) }\end{array}$ & Year & $\begin{array}{l}\text { Representative } \\
\text { ness of the } \\
\text { sample }\end{array}$ & $\begin{array}{l}\text { Justified } \\
\text { sample } \\
\text { size }\end{array}$ & $\begin{array}{l}\text { Response } \\
\text { rate } \\
\text { above } 80 \%\end{array}$ & $\begin{array}{l}\text { Valid } \\
\text { measuremen } \\
t \text { tool }\end{array}$ & $\begin{array}{l}\text { Appropriate } \\
\text { statistical } \\
\text { analysis and } \\
\text { cut-off values }\end{array}$ & $\begin{array}{l}\text { Total } \\
\text { qualit } \\
\text { y } \\
\text { score }\end{array}$ & $\begin{array}{l}\text { Risk of bias } \\
\text { (H- High; L- } \\
\text { Low) }\end{array}$ \\
\hline Ahmad et al. & 2020 & 0 & 1 & 0 & 1 & 1 & 3 & $\mathrm{~L}$ \\
\hline Ahmed et al. & 2020 & 0 & 0 & 0 & 1 & 1 & 2 & $\mathrm{H}$ \\
\hline Amin et al. & 2020 & 0 & 1 & 0 & 1 & 1 & 3 & $\mathrm{~L}$ \\
\hline Banna et al. & 2020 & 0 & 0 & 0 & 1 & 1 & 2 & $\mathrm{H}$ \\
\hline $\begin{array}{l}\text { Chatterjee et } \\
\text { al. }\end{array}$ & 2020 & 0 & 0 & 0 & 1 & 1 & 2 & $\mathrm{H}$ \\
\hline Chew et al. a & 2020 & 0 & 0 & 1 & 1 & 1 & 3 & $\mathrm{~L}$ \\
\hline Chew et al. b & 2020 & 0 & 0 & 1 & 1 & 1 & 3 & $\mathrm{~L}$ \\
\hline Desai et al. & 2020 & 0 & 1 & 0 & 1 & 0 & 2 & $\mathrm{H}$ \\
\hline Grover et al. & 2020 & 0 & 0 & 0 & 1 & 1 & 2 & $\mathrm{H}$ \\
\hline $\begin{array}{l}\text { Gupta AK et } \\
\text { al. }\end{array}$ & 2020 & 0 & 0 & 0 & 1 & 0 & 1 & $\mathrm{H}$ \\
\hline Gupta et al. a & 2020 & 0 & 1 & 0 & 0 & 1 & 2 & $\mathrm{H}$ \\
\hline Gupta et al. b & 2020 & 0 & 1 & 0 & 0 & 1 & 2 & $\mathrm{H}$ \\
\hline Hasan et al. & 2020 & 0 & 0 & 0 & 1 & 1 & 2 & $\mathrm{H}$ \\
\hline Hossain et al. & 2020 & 0 & 0 & 0 & 1 & 1 & 2 & $\mathrm{H}$ \\
\hline Imran et al. a & 2020 & 0 & 1 & 0 & 1 & 1 & 3 & $\mathrm{~L}$ \\
\hline Imran et al. $b$ & 2020 & 0 & 0 & 0 & 1 & 1 & 2 & $\mathrm{H}$ \\
\hline Islam et al. a & 2020 & 0 & 0 & 0 & 1 & 1 & 2 & $\mathrm{H}$ \\
\hline Islam et al. b & 2020 & 0 & 0 & 0 & 1 & 1 & 2 & $\mathrm{H}$ \\
\hline Jain et al. & 2020 & 0 & 1 & 0 & 1 & 1 & 3 & $\mathrm{~L}$ \\
\hline Khanal et al. & 2020 & 0 & 0 & 0 & 1 & 1 & 2 & $\mathrm{H}$ \\
\hline Khanna et al. & 2020 & 0 & 0 & 0 & 1 & 1 & 2 & $\mathrm{H}$ \\
\hline Mamun et al. & 2020 & 0 & 0 & 1 & 1 & 1 & 3 & $\mathrm{~L}$ \\
\hline Mani et al. & 2020 & 0 & 0 & 0 & 1 & 1 & 2 & $\mathrm{H}$ \\
\hline Nisha S et. Al & 2020 & 0 & 0 & 0 & 1 & 1 & 2 & $\mathrm{H}$ \\
\hline $\begin{array}{l}\text { Patabendige } \\
\text { et al. }\end{array}$ & 2020 & 0 & 0 & 0 & 1 & 1 & 2 & $\mathrm{H}$ \\
\hline Reddy et al. & 2020 & 0 & 0 & 0 & 1 & 1 & 2 & $\mathrm{H}$ \\
\hline Sandesh et al. & 2020 & 0 & 0 & 0 & 1 & 1 & 2 & $\mathrm{H}$ \\
\hline $\begin{array}{l}\text { Sebastian et } \\
\text { al. }\end{array}$ & 2020 & 0 & 1 & 0 & 1 & 1 & 3 & $\mathrm{~L}$ \\
\hline Sharma et al. & 2020 & 0 & 0 & 0 & 1 & 1 & 2 & $\mathrm{H}$ \\
\hline Sheshtha et al. & 2020 & 0 & 1 & 0 & 1 & 1 & 3 & $\mathrm{~L}$ \\
\hline Sil et al. & 2020 & 0 & 0 & 0 & 1 & 1 & 2 & $\mathrm{H}$ \\
\hline $\begin{array}{l}\text { Suryavanshi } \\
\text { et al. }\end{array}$ & 2020 & 0 & 0 & 0 & 1 & 1 & 2 & $\mathrm{H}$ \\
\hline Verma et al. & 2020 & 0 & 0 & 1 & 1 & 1 & 3 & $\mathrm{~L}$ \\
\hline Wilson et al. & 2020 & 0 & 1 & 0 & 1 & 1 & 3 & $\mathrm{~L}$ \\
\hline Zubayer et al. & 2020 & 0 & 0 & 0 & 1 & 1 & 2 & $\mathrm{H}$ \\
\hline
\end{tabular}


Supplementary file-B: Meta-regression of studies reporting the prevalence of anxiety

a. Association between the prevalence of anxiety and the percentage of female participants*

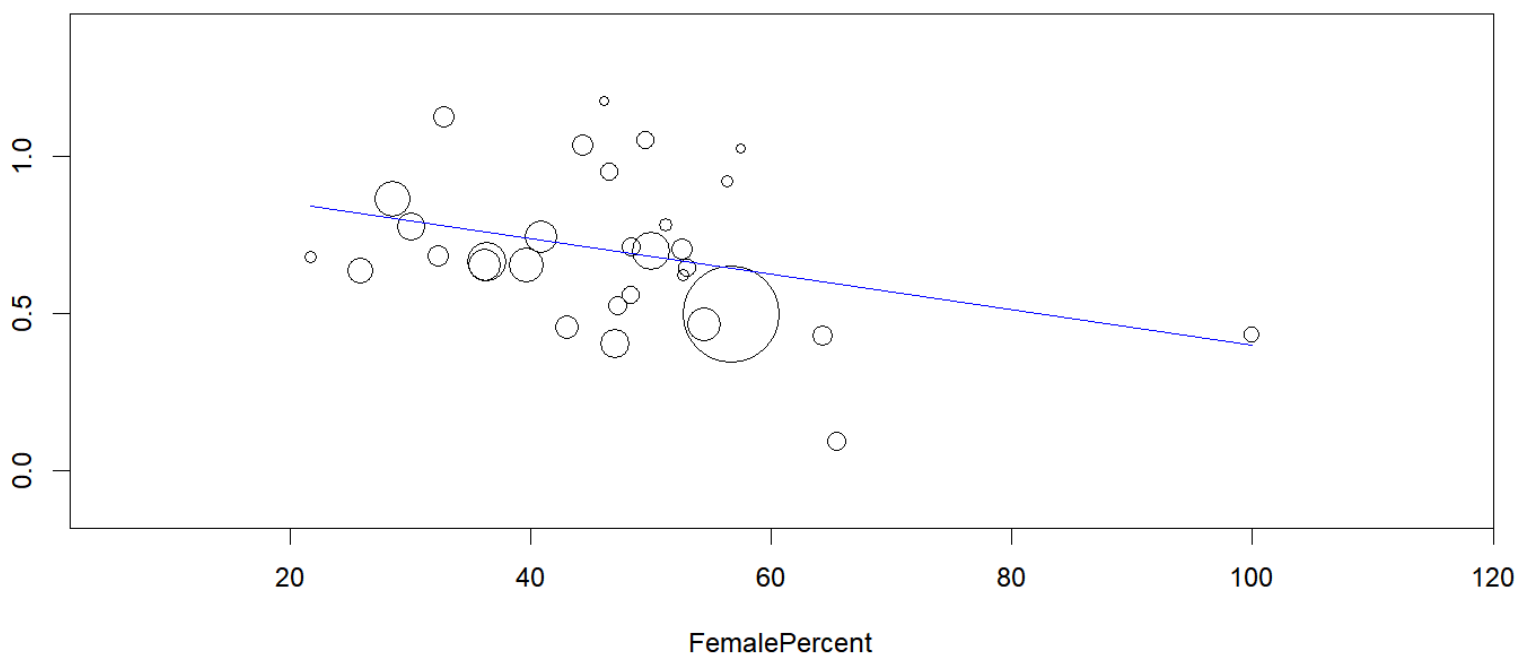

b. Association between the prevalence of anxiety and the mean age of the participants

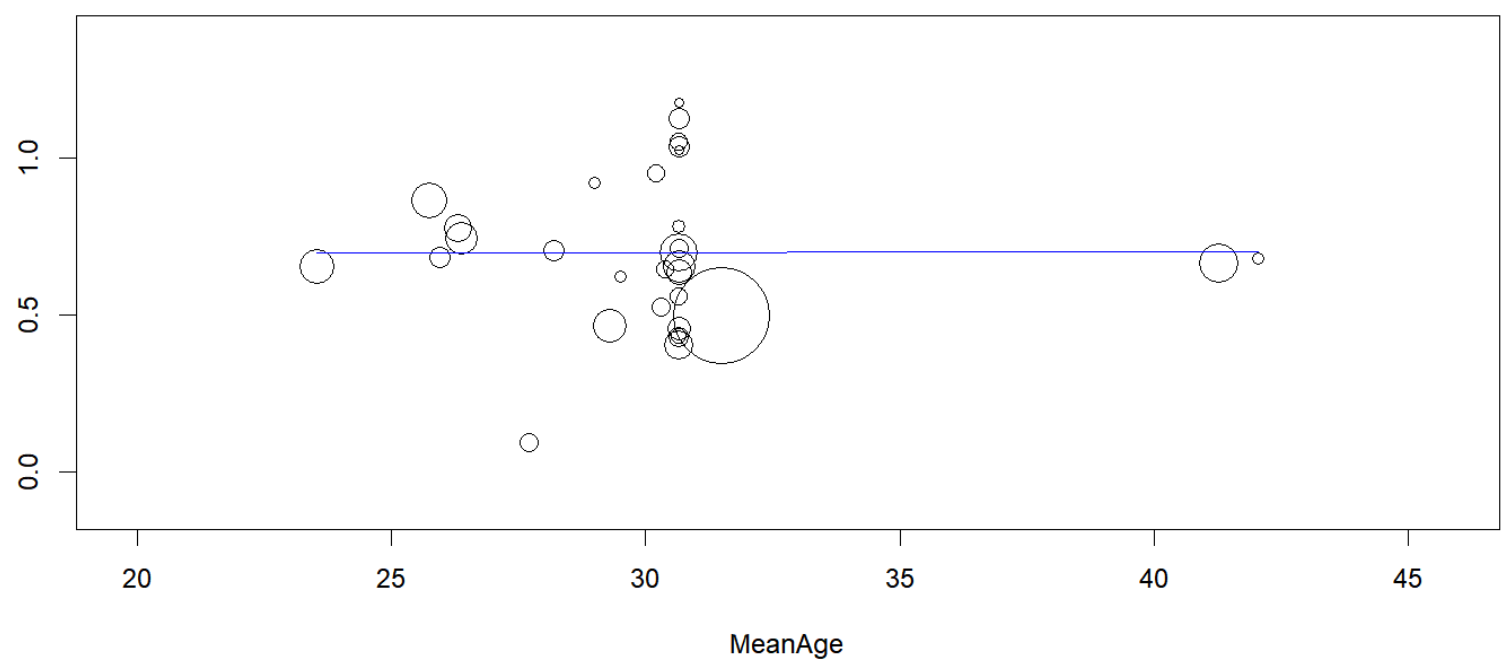


c. Association between the prevalence of anxiety and the quality of score of the studies

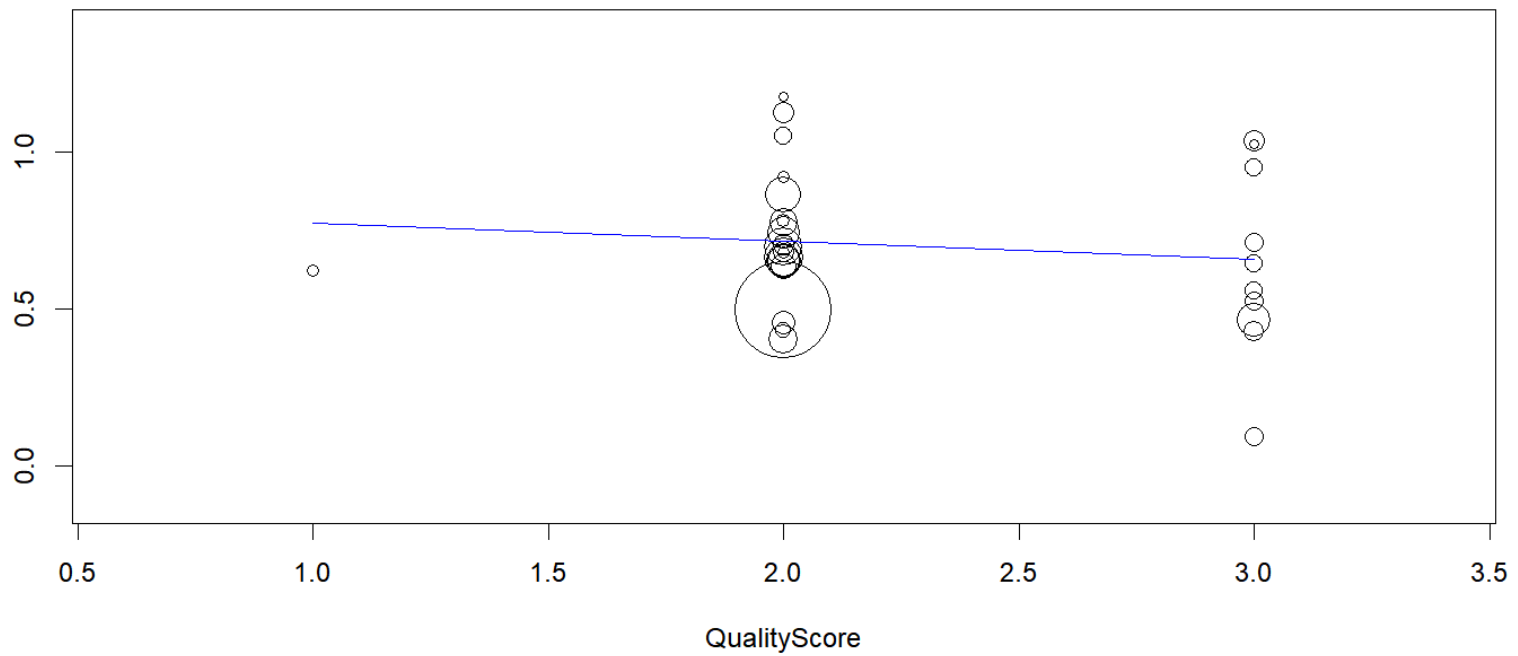

* Statistically significant 
Supplementary file- $C$ : Meta-regression of studies reporting the prevalence of depression

a. Association between the prevalence of depression and the percentage of female participants*

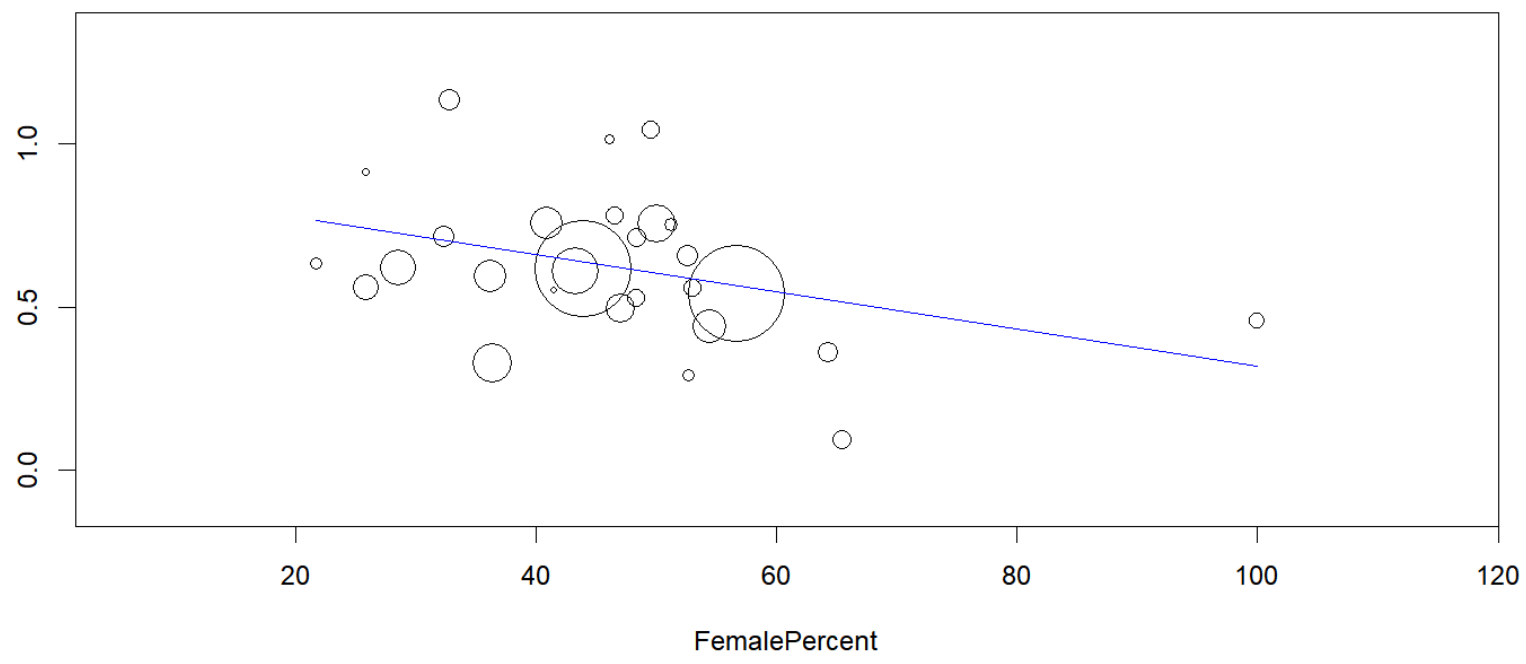

a. Association between the prevalence of depression and the mean age of the participants

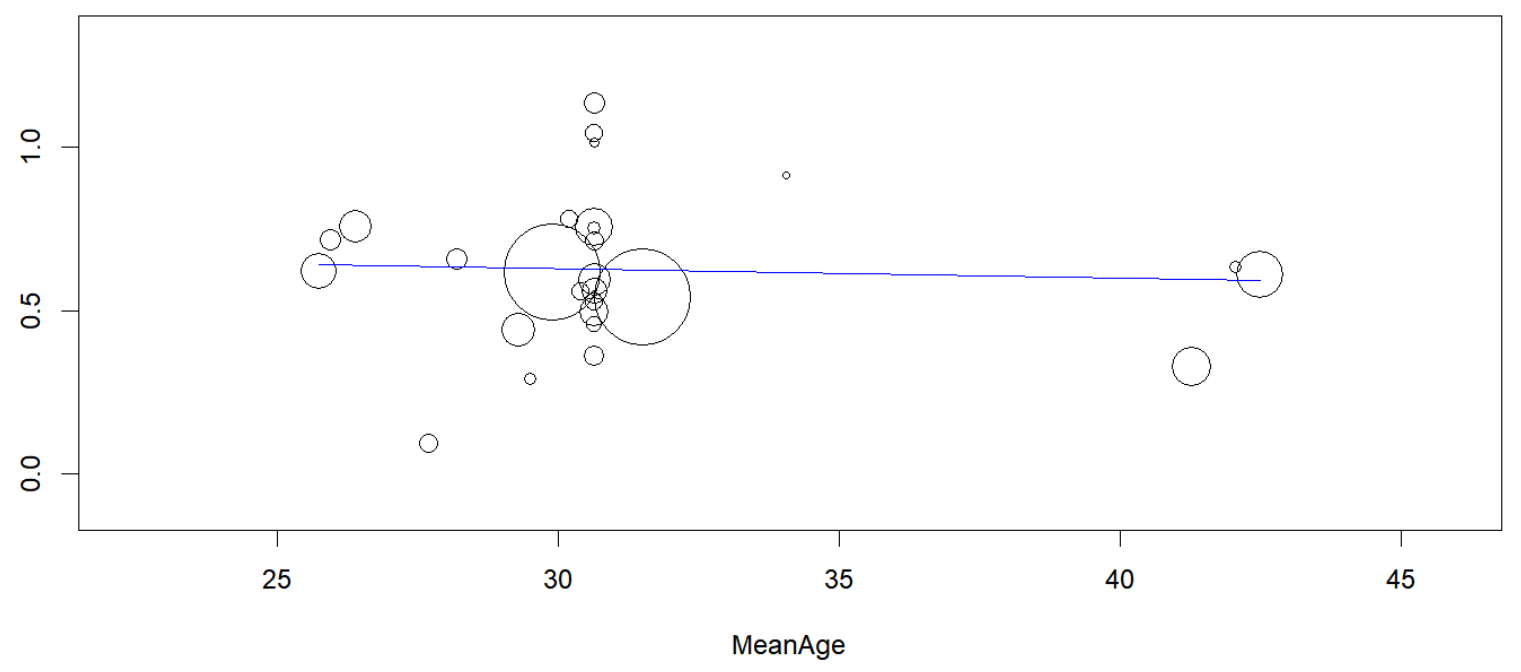


a. Association between the prevalence of depression and the quality of score of the studies

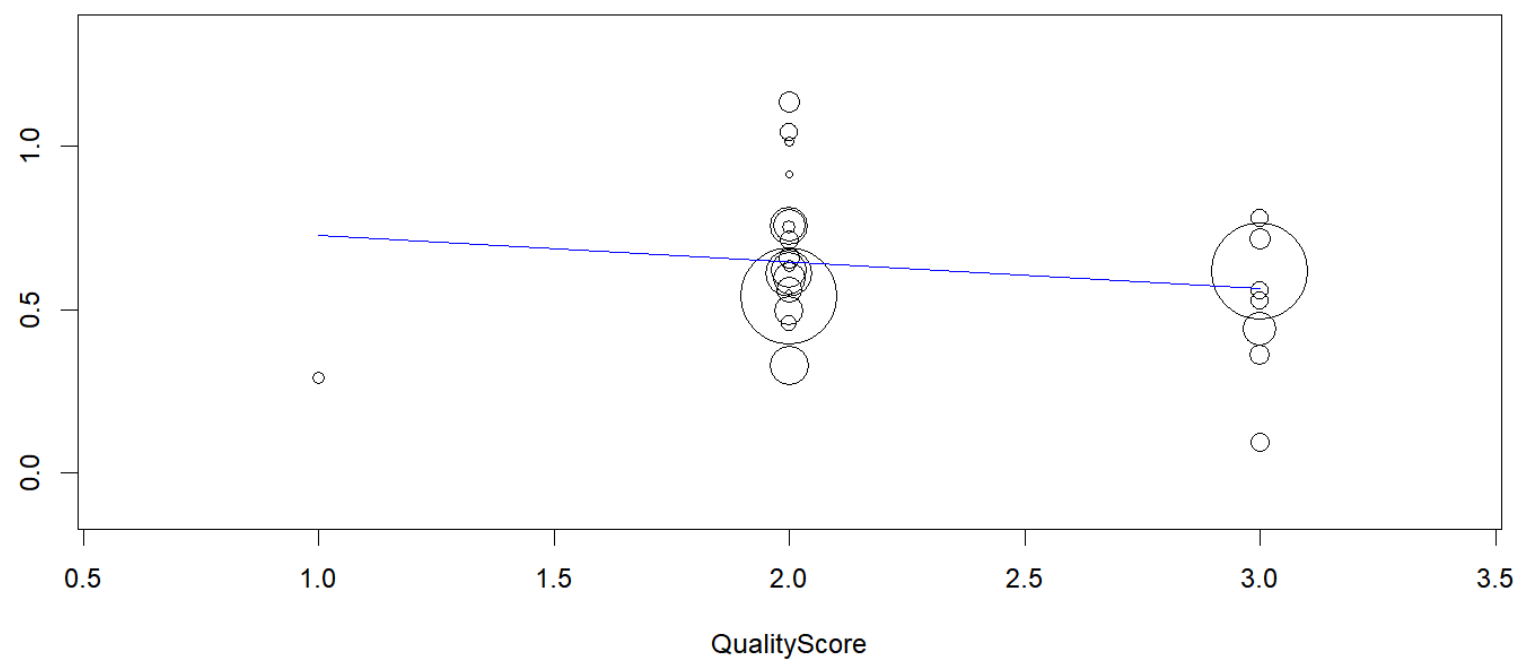

*Statistically significant 